\title{
An Unscented Kalman Filter Based on-line Diagnostic Approach For PEM Fuel Cell Flooding
}

\author{
Xian Zhang ${ }^{1}$, Pierluigi Pisu ${ }^{2}$ \\ ${ }^{1,2}$ Department of Automotive Engineering, Clemson University, Clemson, SC, 29631, USA \\ xianz@g.clemson.edu \\ pisup@clemson.edu
}

\begin{abstract}
Poor water management usually leads to various degrees of flooding in the hydrogen type fuel cell, affecting both the instantaneous performance and the long-term durability of the system adversely. While a lot of fuel cell diagnostic tools exist that could be utilized for the flooding diagnostics, most of these approaches are intrusive, requiring special modification to the fuel cell that affects its integrity, or special equipment (e.g. AC spectrometer) that adds to the complexity and cost of the system, and therefore are not considered to be a viable solution for the on-board integration of the diagnostic scheme.

This paper proposes a model based approach for the fuel cell flooding diagnostics problem, utilizing only the cell current and voltage, and the inlet pressures of the fuel cell as the input signals of the diagnostic scheme. A diagnosticoriented fuel cell system dynamic model is developed to incorporate the effects of the fault, i.e. the flooding, on the system dynamics. For simplicity, only the cathode channel flooding, the cathode gas diffusion layer (GDL) flooding, and the anode channel flooding are considered while we neglect the mass transport loss through the anode GDL. The cathode channel flooding and the GDL flooding diagnostic problems are decoupled and formulated as standard joint state and parameter estimation problems, with the amounts of the liquid water treated as varying system parameters to be identified. The unscented Kalman Filter technique has been applied to solve these problems. Simulation results validate the applicability of the cascading unscented Kalman filter design for flooding diagnostics.
\end{abstract}

\section{INTRODUCTION}

Humidity management is a critical, yet delicate, issue in fuel cell system. While a well-humidified membrane is always

Xian Zhang et al. This is an open-access article distributed under the terms of the Creative Commons Attribution 3.0 United States License, which permits unrestricted use, distribution, and reproduction in any medium, provided the original author and source are credited. desired for good ionic conductivity, excess liquid water accumulation at other locations inside the fuel cell leads to flooding that compromises the performance of the fuel cell. As pointed out in the work by Kumbur et al. (Kumbur, Sharp, \& Mench, 2006), according to different locations of water accumulation, three types of flooding can be identified in PEM fuel cells, namely i) catalyst layer flooding, ii) gas diffusion layer (GDL) flooding, and iii) flow field flooding. Type i) and ii) flooding, which can be further grouped as diffusion media (DM) flooding, are caused by water generation at the reaction site (cathode) or/and water transportation through the membrane. The DM flooding hinders the reactant transport to the catalyst layers where the reaction takes place and results in a higher mass concentration loss, it also aggravates the corrosion and thus the degradation of various of the fuel cell components including the catalyst layer, the GDL, and even the membrane (Zhang, 2012). Type iii) flooding, or channel flooding, is usually caused by water transported through GDL to flow channel under capillary pressure gradient. The liquid water thus entering the channel can reside on the surface of the GDL and also on the other walls of the channel in the form of droplets or water slugs (formed by coalesced droplets) with different sizes. Water accumulated in the channel, and in particular at the interface of the GDL and the channel, also results in higher mass concentration loss; in addition, the cross sectional area of the channel is effectively reduced due to the flow field volume occupation by liquid water, causing higher flow resistance and an increased parasitic pressure loss. Fuel cell flooding diagnosis is, therefore, critically important since the information it provides can be utilized by other on-line supervisory systems to address both the instantaneous performance degradation and the long-term durability issue of the fuel cell systems (Zhang, 2012), e.g. the channel flooding information can be used by some fault-tolerant control system to optimize the purging procedures and mitigate the flooding problem, while the GDL flooding information can be fed to an on-line prognostic and healthmonitoring scheme for system damage tracking and remaining useful life prediction (Zhang \& Pisu, 2012, 
2013). In this paper, the problem of estimation of both channel and GDL flooding is addressed for the first time through the formulation of a joint state and parameter estimation problem.

The fuel cell diagnostic methods in the literature can be roughly grouped into two categories, namely the intrusive method, and the non-intrusive method. The intrusive diagnostic method usually requires special modification to the fuel cell that affects its integrity (e.g., fuel cell with transparent bipolar plates for channel flooding observation), or alters the fuel cell's operating conditions (e.g., EIS method that imposes a perturbation current or voltage on the fuel cell). The non-intrusive diagnostic method, on the other hand, utilizes only information from the plant sensors while keeping the fuel cell's integrity and does not change the system's normal operating conditions.

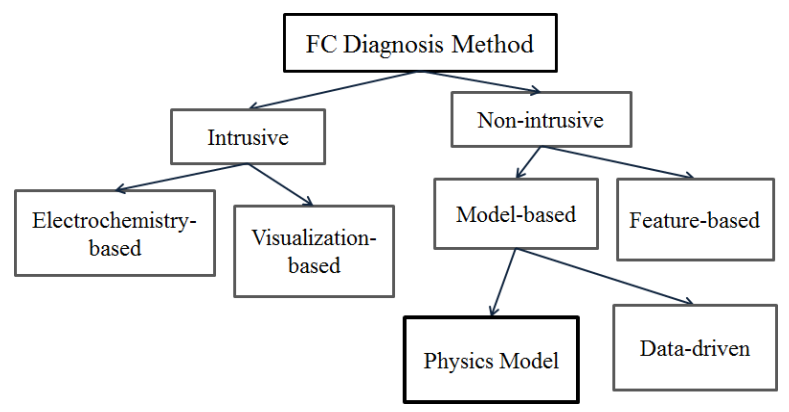

Figure 1. A classification of fuel cell (FC) diagnosis method

In the intrusive method category, two subcategories are usually seen, one is the so-called visualization-based method such as neutron imaging techniques and the aforementioned transparent fuel cell; the other is the electrochemistry-based method such as the Electrochemical Impedance Spectroscopy (EIS) techniques and cyclic voltammetry.

The most widely applied electrochemical-based diagnostics tool is the EIS technique, which is used to measure the fuel cell's AC impedance around some steady state operating point as a function of frequency (Fouquet, Doulet, Nouillant, Dauphin-Tanguy, \& Ould-Bouamama, 2006; Gazzarri, Eikerling, Qianpu Wang, \& Zhong-Sheng Liu, 2010; Gebregergis, Pillay, \& Rengaswamy, 2010; Hoshiko, Nakajima, Konomi, Kitahara, \& Kita, 2008; Le Canut, Abouatallah, \& Harrington, 2006; Makharia, Mathias, \& Baker, 2005; Narjiss, Depernet, Candusso, Gustin, \& Hissel, 2008; Wasterlain, Harel, Candusso, Hissel, \& Francois, 2009; Yuan, Sun, Wang, \& Zhang, 2006). The basic idea of the EIS-based diagnosis is to exploit the different impacts of different faults (e.g., water flooding and membrane drying) on the measured impedance (in terms of magnitude and phase) to detect and differentiate the faults. Equivalent circuit models of the fuel cell are often built for data fitting of the measured fuel cell impedance, and the fitted parameters that are sensitive to some faults can then be used as indicators of the faults (e.g., (Fouquet et al., 2006)). Although the EIS technique (or an extension of this method known as nonlinear frequency response analysis (Kadyk, Hanke-Rauschenbach, \& Sundmacher, 2009) provides resourceful information about the operating conditions of the fuel cell, the measurement of the AC impedance usually requires maintained steady state operating conditions (steady voltage or current) during the whole frequency sweep session, and sometimes perturbation current with large magnitude, which is quite stringent especially for the fuel cell with automotive application. Also, the use of an $\mathrm{AC}$ spectrometer is not a very convenient solution for onboard integration due to the complexity and cost of the equipment. For a more comprehensive review of the electrochemical techniques applied to the fuel cell system, the interested readers could refer to the review papers (Xiaozi Yuan, Haijiang Wang, Jian Colin Sun, \& Jiujun Zhang, 2007)(Wu et al., 2008).

Non-intrusive method can be further divided into two subcategories, namely the model based diagnostic method and the feature based diagnostic method. Feature based methods do not rely on any form of models, rather, they extract features that are sensitive to certain faults from the external measurable variables such as voltage and pressure drop across the fuel cell channel, and analyzes the "symptom" in the feature, which is usually acquired as a prior knowledge from experimental data, for the fault detection and isolation. Steiner et al. (N.Y. Steiner, Hissel, Mocoteguy, \& Candusso, 2011) proposed a wavelet analysis method that, utilizing only the stack voltage signal, allows the detection of the flooding based on the feature patterns obtained from the wavelet packet coefficients. Similarly, Niroumand et al. (Niroumand, Merida, \& Saif, 2011) used cell voltage oscillation to isolate cathode flooding.

Pressure drop is a feature, or physical indicator, that has been widely used for the channel flooding. Chen and Zhou (Chen \& Zhou, 2008) describes an approach to utilize frequency of pressure drop signal as a diagnostic tool for PEM fuel cell stack dynamic behaviors. Barbir et al. (Barbir, Gorgun, \& Wang, 2005) diagnose flooding and drying conditions inside a stack by monitoring pressure drop and directly measuring cell resistance respectively in an operational fuel cell stack. General Motors patented a method and apparatus that was based on pressure drop monitoring for detecting and correcting water flooding in an air-breathing PEM fuel cell (Bosco \& Fronk, 2000) by comparing the measured pressure drop across flow field of a fuel cell stack to acceptable pressure drops (in a lookup table) determined empirically from a substantially identical, unflooded stack at various electrical discharge rates. Pei et al.(Pei et al., 2006) showed that the hydrogen pressure drop is strongly affected by liquid water content in the flow channel of fuel cells, and it is not in normal relation with flow rate when the stoichiometric ratio is varied. The total pressure drop can be calculated by a frictional pressure loss 
formula accurately, relating with mixture viscosity, stack temperature, operating pressure, stoichiometric ratio and stack current.

The feature-based method provides little to none physical insight into the fault nature. And due to the limited available features and the fact that one feature (e.g. the voltage) can usually be affected by multiple possible faults (e.g. flooding, drying, catalyst poisoning), the faults that can be detected and identified by the chosen feature are also limited. To achieve the fault detection and identification at the same time, model information of the fuel cell needs to be exploited.

Data driven models, such as fuzzy logic and neural network models, have found their application in the fuel cell diagnostics. For example, in (Hissel, Péra, \& Kauffmann, 2004) a fuzzy diagnostic-oriented static fuel cell model with the voltage and current as the model input is proposed. In (N. Yousfi Steiner, Candusso, Hissel, \& Mooteguy, 2010), Steiner et al. presented a flooding diagnosis procedure based on black-box model, The flooding diagnosis procedure is based on the analysis of a residual obtained from the comparison between an experimental and an estimated pressure drop. The estimation of this latter is ensured by an artificial Neural Network that has been trained with flooding-free data.

Physics model based approaches have the advantage of better portability and scalability. However, due to the extremely complex physical structure of fuel cells and the nature of weakly coupled internal states, which could lead to observability problem, there are only a few studies focusing on the estimation or fault diagnosis problems based on first principle fuel cell models, including those with application of observer techniques to water related state estimation of PEMFC. Arcak et al. (Arcak, Gorgun, Pedersen, \& Varigonda, 2004) designed an adaptive observer to estimate the partial pressure of hydrogen in the anode channel of a fuel cell by assuming the inlet partial pressure is slowly varying and treating it as an unknown parameter. Aitouche et al. (Aitouche et al., 2011) defined two actuator faults and two sensor faults and applied a parity space approach with nonlinear analytical redundancy to address the fault detection and isolation problem. Gorgun et al. (Gorgun, Arcak, \& Barbir, 2006) proposed an estimation scheme for PEMFC membrane humidity by exploiting its effect on cell resistive voltage. The scheme can detect membrane drying condition but lack the ability to distinguish between drying and flooding inside the fuel cell. McKay and Stephanopoulou (D. McKay \& Stefanopoulou, 2004) presented a model with lumped membrane diffusion parameter for estimating the electrode humidity of a PEMFC stack. Based on this model, the authors developed a nonlinear open loop estimator of the membrane humidity by utilizing the pressure and temperature entering and exiting the electrodes, as well as the upstream humidity of each electrode. In (Vepa, 2012), Vepa applied a unscented Kalman filter with the ability to estimate and adapt the process noise covariance matrix to the state estimation of a PEMFC by utilizing measurement of the stack voltage and current, the stack temperature, and the relative humidity in the electrodes. The designs of both the last two observers require humidity sensors, which limit their real-time control application due to their cost, size, and inadequate response time and accuracy (Gorgun et al., 2006). Also, none of the models employed in the above estimation schemes have taken the GDL module into consideration, thus lack the ability to detect the GDL flooding.

In this paper, a physics model based approach is proposed for the fuel cell flooding diagnostics in the channels and in the cathode GDL. A control-oriented fuel cell system dynamic model that incorporates the flooding effect on the fuel cell system outputs is proposed, taking into account of both GDL flooding and channel flooding. (It should be pointed out that the fuel cell outputs and performances would also be affected by slow degradation of the fuel cell components in the long run. In (Zhang \& Pisu, 2012), a prognostic problem is studied with the long-term cathode catalyst degradation being the focus of that paper. However, since the time constants of these two problems are orders of magnitude apart (second for the diagnostic problem, and tens of hours for the prognostic problem), the two problems can be well treated as independent. Thus in this paper, fuel cell aging effect is not considered and only the instantaneous fault effect on the system performance is investigated.) The amounts of the liquid water in both the channel and the GDL are treated as varying system parameters (or unknown disturbances) to be identified, and modeled as stochastic processes driven by white noises. To make the problem an observable one and considering that the cathode GDL flooding has a much greater effect on the cell voltage than the same level anode GDL flooding (this conclusion can be obtained from detailed model analysis), only cathode GDL flooding is considered in this paper while we assume no mass transport loss at anode. The cathode channel flooding diagnostic problem is then decoupled from the GDL flooding diagnostic problem, and the resulting two problems are both formulated as standard simultaneous state and parameter estimation problem, with the liquid water volumes in the channel and the GDL treated as varying system parameters. Considering the highly nonlinear and stochastic nature of the plant, nonlinear optimal state estimation techniques, such as the nonlinear versions of the Kalman filters, are deemed to be an optimum choice to solve the problem. A comparison of stochastic filtering techniques shows that, the extended Kalman filter (EKF) is often difficult to tune, and can only handle a limited amount of nonlinearity, while the particle filtering (PF) usually imposes high computational complexity(Wan \& Merwe, 2001). In this paper, the unscented Kalman Filter (UKF) technique is selected for the implementation of the 
estimation framework presented in Section 4 because of the reasonable trade-off between computational complexity and capability of handling system nonlinearities.

The paper is organized as follows: Section 2 presents the structure of the fuel cell system under study and a formal statement of the diagnostic problem to be solved; in Section 3 , a diagnostic-oriented model is developed for the fuel cell system, with the focus on the gas supplying subsystem and the GDL module at the cathode side; Section 4 gives a brief introduction to the general UKF framework for joint state/parameter estimation, based on which a diagnostic scheme is designed in Section 5 for the fuel cell flooding diagnostic problem; finally in Section 6, simulation is carried out with dictated water volumes in both the channel and the GDL of the fuel cell cathode to validate the diagnostics design.

\section{Problem Formulation}

A PEM fuel cell system is considered in this paper with flow-through reactant supply systems on both anode and cathode sides. Figure 2 shows a simplified diagram of the fuel cell system, with the air supply system accentuated that supplies air to the fuel cell cathode flow channel to replenish the oxygen being consumed and maintains the reactant gas pressure at the electrode at some desired level. The $\mathrm{H}_{2}$ supply system is omitted in the diagram due to the symmetric structure of the system. The reactant supply subsystem is composed of a mass flow controller (MFC), a gas supplying pipeline (supply manifold in the case of a commercial fuel cell stack), a humidifier (water injection in the diagram), fuel cell flow channel(s), a return pipeline and a back pressure regulator.

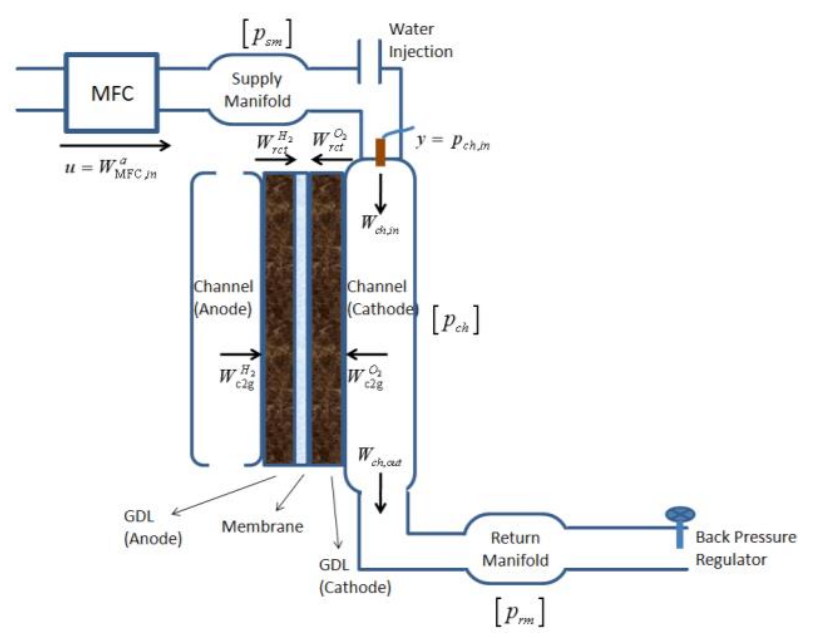

Figure 2 Simplified diagram of the fuel cell system

As depicted in the above picture, the mass flow controller (MFC) acts as the subsystem's actuator and controls the mass flow rate of the inlet air $\left(W_{\mathrm{MFC}, \text { in }}^{a}\right)$ entering the air supply system. The incoming air is conducted by the supplying pipeline to the humidifier to receive humidification before entering the fuel cell channel, where part of the oxygen is diffused into and distributed by the GDL (with a mass flow rate of $W_{\mathrm{c} 2 \mathrm{~g}}^{O_{2}}$ ) to the catalyst site for reaction (with a reaction rate of $W_{\text {rct }}^{O_{2}}$ ). The remainder of the air and produced water vapor then exits the fuel cell channel and is conducted by the returning pipeline to leave the air supplying system and enter the atmosphere. The manually adjustable back pressure regulator is located at the end of the return pipeline to operate the fuel cell at the specified pressure, and the fuel cell channel inlet pressure is measured by the pressure sensor installed immediately before the fuel cell inlet.

The problem considered in this paper can be stated as follows:

Construct a diagnostic scheme that utilizes the available inputs and outputs of the fuel cell system as described above to generate a signal (residual) that can effectively indicate the existence and magnitude of the considered system faults, which are water flooding inside the flow fields and the GDLs of the fuel cell.

\section{A Diagnostic-oriented Fuel Cell Model}

Current control-oriented fuel cell models, though computationally efficient and capable of capturing the fuel cell dynamics, do not account for fault effect on the system and are not suitable to be directly utilized in a diagnostic scheme. For diagnosis or estimation purpose, a fuel cell model is desired that incorporates the fault (in this case, water flooding inside the fuel cell) effects on the system dynamics, as well as the system outputs.

This section presents a diagnostic-oriented dynamic fuel cell model, which is mainly composed of two subsystems (on either anode or cathode side of the fuel cell), i.e. reactant supplying subsystem, and gas diffusion subsystem. For illustration, we only focus on the development of the model of the fuel cell cathode side. The model on the anode side can be derived in a similar fashion.

\subsection{Model assumption}

We first lay out the following model assumptions as the basis for the model development:

1. Both cathode and anode sides of the fuel cell are openended;

2. All the gases obey the ideal gas law;

3. The humidifier fully humidifies the inlet reactant;

4. There is no liquid water flowing into the fuel cell flow channel (no flooding before fuel cell);

5. The reactant gas flowing at the cathode (humidified air) is a unified mixture (constant molar mass); 
6. The oxygen mass fraction flowing out of the channel is equal to that in the channel;

7. The fuel cell is largely operating under constant temperature in isothermal condition;

8. No $\mathrm{N}_{2}$ or water vapor is transported across the GDL.

\subsection{Fuel cell air supply system model}

For a general pipe filling with flowing fluid, the frictional pressure drop at steady state can be represented as (O’Hayre, Cha, Colella, \& Prinz, 2009)

$$
\frac{d p}{d x}=\frac{2 \chi \cdot \bar{u} \cdot \mu}{D_{h}^{2}}
$$

where $p$ is the pressure at $x$ (the distance from the channel inlet), $\chi$ is a dimensionless constant depending on the geometry of the pipe cross section (for circular channels $\chi=16), D_{h}$ is the hydraulic diameter of the fuel cell flow channel $(\mathrm{m}), \mu$ is the fluid viscosity $(\mathrm{kg} / \mathrm{m} \cdot \mathrm{s}) . \bar{u}$ is the mean flow velocity of the fluid $(\mathrm{m} / \mathrm{s})$. In terms of mass flow rate $W(\mathrm{~kg} / \mathrm{s}), \bar{u}=\frac{W}{A \cdot \rho}$, where $A$ is the cross sectional area of the channel $\left(\mathrm{m}^{2}\right)$, and $\rho$ is the fluid density $\left(\mathrm{kg} / \mathrm{m}^{3}\right)$ at $(x, t)$, the partial derivative of which w.r.t. time is

$$
\frac{\partial \rho}{\partial t}=-\frac{1}{A} \frac{\partial W}{\partial x}
$$

For ideal gas, $p=\frac{\rho}{M} R T$, with $M$ being the molar mass of the flowing gas $(\mathrm{kg} / \mathrm{mol}), R$ being the universal gas constant $(\mathrm{J} / \mathrm{mol} \cdot \mathrm{K})$ and $T$ being the absolute temperature $(\mathrm{K})$. Substituting the ideal gas law in (1) and (2) results in

$$
\begin{gathered}
\frac{\partial p}{\partial t}\left(\frac{A M}{R T}\right)=-\frac{\partial W}{\partial x} \\
-\frac{\partial p}{\partial x}=k W(x)
\end{gathered}
$$

where $k$ can be calculated as $k=\frac{2 \chi \cdot \mu(T)}{D_{h}^{2} \cdot A \cdot \rho}=\frac{2 \chi \cdot \mu(T) \cdot R \cdot T}{D_{h}^{2} \cdot A \cdot M \cdot p}$, here $\mu$ is explicitly expressed as a function of the temperature. As in fuel cell flow gas streams are almost always gas mixture (e.g. in cathode side of PEMFC, the gas mixture is composed of oxygen, nitrogen and water vapor) instead of pure gas, the viscosity of the gas mixture would then dependent on the components of the mixture, as well as the mixture's absolute temperature.

While the analytical solution to the above PDEs is hard to obtain, and precise modeling may require detailed finite element analysis, whose numerical solution imposes high computational burden, a lumped model is considered in this paper to suit the diagnostic-oriented need that inherently entails reduced model complexity.

Consider again the general pipe with two open ends, with the lumped pressure instead of the distributed one as function of $x$, and discretize the right hand side of the equation at the pipe inlet and outlet, equation (3) can be written as

$$
\frac{d p}{d t}\left(\frac{A M}{R T}\right)=\frac{W_{\text {in }}-W_{\text {out }}}{x_{\text {out }}-x_{\text {in }}}=\frac{W_{\text {in }}-W_{\text {out }}}{L}
$$

where $L$ is the pipe length, $W_{\text {in }}$ and $W_{\text {out }}$ are the mass flow rate of the flowing fluid at the pipe inlet and outlet, respectively. By applying the ideal gas law to equation (5), it can be easily shown that (5) has essentially the same form as that derived from mass conservation law.

Now, rewrite (4) as $-d p=k W(x) d x$ and take the integration of both sides of the equation while assuming identical $k$ and $W(x)$ through the pipe length (lumped model), we have

$$
W=-\frac{\Delta p}{k \Delta x}
$$

which is applicable to fluid flow inside the pipe.

On the other hand, the relation between pressure drops at junctions of pipes or nozzles and the local mass flow rates could be approximated linearly by introducing a frictional nozzle constant $K_{j}$ ( $j=i n$ for inlet nozzle constant, and $j=$ out for outlet nozzle constant), i.e. $\Delta p=K_{j} W$, when the pressure drop is relatively small.

By applying (5) and (6) to the three separate volumes of the fuel cell air supply system (Figure 2), i.e., lumped supplying manifold, channel volume, and return manifold, the following state space equations describing the gas dynamics in the system is obtained:

$$
\begin{gathered}
\frac{M V_{s m}}{R T} \dot{p}_{s m}=W_{\text {in }}-\frac{p_{s m}-p_{c h}}{K_{s m, \text { out }}+K_{c h, \text { in }}+\frac{k_{c h} L}{2}} \\
\frac{M V_{c h}}{R T} \dot{p}_{c h}=\frac{p_{s m}-p_{c h}}{K_{s m, \text { out }}+K_{c h, \text { in }}+\frac{k_{c h} L}{2}}-W_{c 2 g}-\frac{p_{c h}-p_{r m}}{\frac{k_{c h} L}{2}+K_{c h, \text { out }}+K_{r m, \text { in }}} \\
\frac{M V_{r m}}{R T} \dot{p}_{r m}=\frac{p_{c h}-p_{r m}}{\frac{k_{c h} L}{2}+K_{c h, \text { out }}+K_{r m, \text { in }}}-\frac{p_{r m}-p_{b p r}}{K_{r m, \text { out }}}
\end{gathered}
$$

where $W_{i n}=W_{i n}^{a}+W_{h m}^{v}$ is the sum of the mass flow rate of the inlet air controlled by the mass flow controller and the water 
vapor provided by the ideal humidifier, while $W_{\mathrm{c} 2 \mathrm{~g}}$ is the total mass flow rate flowing from the channel to the GDL.

For the simplicity of illustration and to gain further physical insight to the gas supplying system, an analogy to an electrical ciruit (Danzer, Wilhelm, Aschemann, \& Hofer, 2008) of the above state space model is considered and shown in Figure 3, where pressures are represented by voltages and mass flow rates by currents.

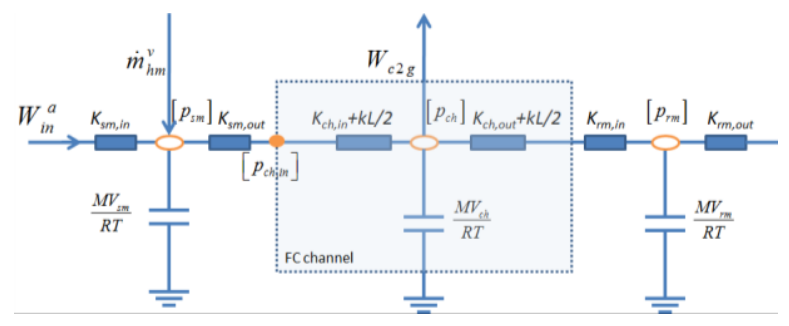

Figure 3 A simplified electrical circuit model for air supplying system

According to Figure 3, the pressure at channel inlet can be easily obtained as

$$
p_{c h, i n}=p_{c h}+\left(\frac{p_{s m}-p_{c h}}{K_{s m, o u t}+K_{c h, i n}+\frac{k_{c h} L}{2}}-W_{c 2 g}\right) \times\left(K_{c h, i n}+\frac{k_{c h} L}{2}\right)
$$

This completes the derivation of the full lumped model for the air supply system, which includes the effect of the channel frictional coefficient on the gas dynamics and the inlet pressure as well.

\subsection{Gas diffusion model in the cathode GDL}

A general GDL model for the purpose of system analysis should address the flow dynamics of various species in the GDL, including - for the case of the cathode GDL in a PEMFC $-\mathrm{O}_{2}, \mathrm{~N}_{2}$, water vapor and liquid water. Such detailed model can be found in the literatures like (D.A. McKay, Ott, \& Stefanopoulou, 2005) or (Denise A. McKay, Siegel, Ott, \& Stefanopoulou, 2008), where the GDL is discretized in three volumes. In this paper, to minimize the model complexity and facilitate the design of the diagnostic scheme, only $\mathrm{O}_{2}$ diffusion in the GDL is modeled by considering the GDL as a lumped volume. The liquid water in the GDL, similar to that in the fuel cell channel, is treated as an unknown, varying parameter to be identified by the diagnostic scheme.

Figure 4 shows two scenarios of $\mathrm{O}_{2}$ transport in the GDL with different humidity levels to qualitatively illustrate the effect of the GDL liquid water on the $\mathrm{O}_{2}$ diffusion. In each scenario, the depicted three volumes are respectively, from left to right, the membrane, the GDL, and the channel. For illustration purpose, the geometries (widths) of the three volumes are out of proportion.
As demonstrated in Figure 4, water flooding hinders the gas transport in the GDL and results in a larger pressure difference $\left(p_{c h}^{O_{2}}-p^{* O_{2}}\right)$ across the GDL thickness, thus a larger mass transport loss and a smaller $\mathrm{O}_{2}$ partial pressures at the reaction site $\left(p^{* O_{2}}\right)$. As this looks similar to the situation in the flow field, note there is a difference in the mechanism of the two types of gas transport: while the gas flow in the channel is a convective flow driven by the bulk pressure (total pressure) difference, in the gas diffusion media (DM) it is a diffusivity flow of a certain gas species that is driven by its own concentration (partial pressure) gradient w.r.t. space.

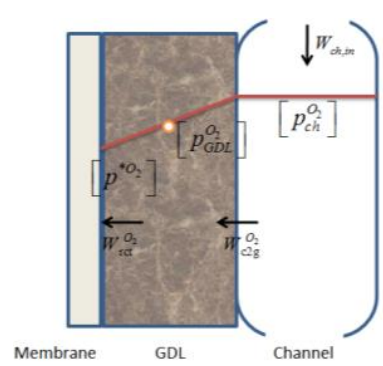

(a) Dry condition

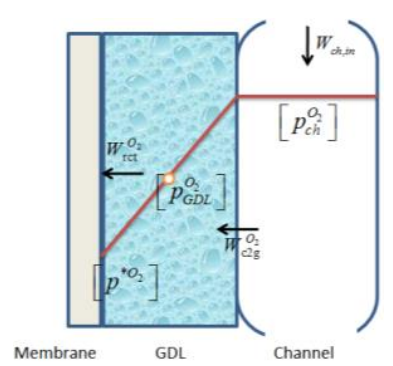

(b) Flooding condition
Figure $4 \mathrm{O}_{2}$ partial pressure profile at the cathode of PEMFC at steady state

The local diffusion rate in molar flux $\left(\mathrm{mol} / \mathrm{m}^{2} \cdot \mathrm{s}\right)$ is given by (Denise A. McKay et al., 2008) as follows

$$
N_{\delta}=-\left\langle D_{\text {eff }}^{O_{2}}\right\rangle \frac{\partial c_{\delta}^{O_{2}}}{\partial \delta}
$$

where $\delta$ is the depth into the GDL from the GDL/channel interface, $c_{\delta}^{O_{2}}$ is the concentration of $\mathrm{O}_{2}$ at $\delta$, and $\left\langle D_{\text {eff }}^{O_{2}}\right\rangle$ is the effective diffusion coefficient for $\mathrm{O}_{2}$ at $\delta$, which is affected by the material of the diffusion media, and the flooding level in the GDL as well. At steady state, assuming the flooding water is evenly distributed across the GDL thickness, or equivalently, assuming that $\left\langle D_{\text {eff }}^{O_{2}}\right\rangle$ is the same at any $\delta$, the diffusion rate $N_{\delta}$ is identical everywhere in the GDL implies identical concentration gradient $\frac{\partial c_{\delta}^{O_{2}}}{\partial \delta}$ everywhere in the GDL, thus the linear profile of the $\mathrm{O}_{2}$ partial pressure in Figure 4 (since the concentration of a gas species is proportional to its partial pressure, i.e., for $\mathrm{O}_{2}$ we have $\left.c_{\delta}^{O_{2}}=\frac{p_{\delta}^{O_{2}}}{R T}\right)$

To obtain the lumped model, consider a single state variable $p_{G D L}^{O_{2}}$ to represent the $\mathrm{O}_{2}$ partial pressure in the GDL volume as a whole (refer to Figure 4). Denote the mass of 
the oxygen gas in the GDL as $m_{G D L}^{O_{2}}$, and apply again the mass conservation law, we have

$$
\dot{m}_{G D L}^{O_{2}}=W_{\mathrm{c} 2 \mathrm{~g}}-W_{r c t}^{O_{2}}
$$

The left hand side of the above equation can be written as $\dot{m}_{G D L}^{O_{2}}=\dot{p}_{G D L}^{O_{2}} \frac{V_{G D L}^{\text {pore }} \cdot M_{O_{2}}}{R T}$ according to the ideal gas law. And from assumption 8, the total mass flow rate transporting from the channel into the GDL can be represented by (13)

$$
W_{\mathrm{c} 2 \mathrm{~g}}=W_{\mathrm{c} 2 \mathrm{~g}}^{O_{2}}=D_{\mathrm{eff}}^{O_{2}}\left(V_{G D L}^{l}\right) \cdot \frac{c_{c h}^{O_{2}}-c_{G D L}^{O_{2}}}{\delta_{G D L} / 2} M_{O_{2}} \cdot A_{\mathrm{fc}}
$$

where $D_{\text {eff }}^{O_{2}}(\bullet)$ is a function of the volume of the liquid water contained in the GDL that outputs the effective diffusivity specific to $\mathrm{O}_{2}$ in the GDL, and is given in (Denise A. McKay et al., 2008) as follows

$$
D_{\text {eff }}^{O_{2}}\left(V_{G D L}^{l}\right)=D^{O_{2}} \varepsilon\left(\frac{\varepsilon-0.11}{1-0.11}\right)^{0.785}\left(1-\frac{V_{G D L}^{l}}{V_{\text {pore }}}\right)^{2}
$$

where $D^{O_{2}}$ is the gas diffusion coefficient for $\mathrm{O}_{2}, \varepsilon=\frac{V_{\text {pore }}}{V_{G D L}}$ is the GDL porosity, which is the ratio between the pore volume and bulk volume of the GDL.

Substitute $\dot{m}_{G D L}^{O_{2}}=\dot{p}_{G D L}^{O_{2}} \frac{V_{G D L}^{p o r e} \cdot M_{O_{2}}}{R T}$ and equation (13) in (12) , we finally get the state equation describing the dynamics of the $\mathrm{O}_{2}$ concentration inside the GDL, as given below

$$
V_{G D L}^{\text {pore }} \dot{p}_{G D L}^{O_{2}}=D_{\text {eff }}^{O_{2}}\left(V_{G D L}^{l}\right) \cdot \frac{p_{c h}^{O_{2}}-p_{G D L}^{O_{2}}}{\delta_{G D L} / 2} \cdot A_{\mathrm{fc}}-\frac{W_{r c t}^{O_{2}}}{M_{O_{2}}} R T
$$

The $\mathrm{O}_{2}$ partial pressures at the reaction site can then be represented as

$$
p^{* O_{2}}=p_{G D L}^{O_{2}}-W_{r c t}^{O_{2}} \frac{\delta_{G D L} / 2 \cdot R T}{M_{O_{2}} A_{\mathrm{fc}} D_{\text {eff }}\left(V_{G D L}^{l}\right)}
$$

which is used to calculate the cell voltage in the voltage module to be presented in the next subsection.

\subsection{Cell voltage model}

The cell voltage under operation is the difference of the ideal voltage and various kinds of overpotentials, as described in the following equation

$$
V_{\text {cell }}=E-\eta_{a c t}-\eta_{\text {ohm }}
$$

where $E$ is the reversible cell voltage, which is the ideal voltage a fuel cell can produce by thermodynamic theory; $\eta_{\text {act }}$ is the overpotential due to activation loss; $\eta_{\text {ohm }}$ is the overpotential due to Ohmic loss.

Generally, in form, the overpotentials for electrochemical systems like fuel cells also include overpotential due to mass transport loss (or concentration loss overpotential $\eta_{\text {conc }}$ ) in addition to that of the activation loss and Ohmic loss, as seen in most literature. The inclusion of this term accounts for the concentration loss during mass transport when using operating conditions in the flow field instead of that at catalyst sites in cell voltage calculation. However, when mass transport component model (mainly the GDL module) is incorporated in the fuel cell model to account for the concentration loss and the operating conditions at the reaction sites can be directly obtained and used, the term $\eta_{c o n c}$ should be left out of the cell voltage equation to avoid double counting. We explain this idea in more detail as we describe each component part of the cell voltage individually in the following.

\subsubsection{Reversible cell voltage}

For hydrogen fuel cell, the reversible cell voltage is given by

$$
E=1.229-0.85 \times 10^{-3}\left(T_{f c}-298.15\right)+\frac{R T_{f c}}{2 F} \ln \left[\frac{p_{H_{2}}^{*}\left(p_{O_{2}}^{*}\right)^{\frac{1}{2}}}{p_{H_{2} O}}\right]
$$

where $p_{\mathrm{H}_{2}}^{*}$ and $p_{\mathrm{O}_{2}}^{*}$ are reactant partial pressures at catalyst layer, expressed in atm. Note in (Pukrushpan, Peng, \& Stefanopoulou, 2004) and other literature where concentration loss overpotential is included, the pressures in (18) are those at flow field instead of reaction sites. We use "** to denote variables at catalyst layer in the following for emphasis.

\subsubsection{Activation loss}

Activation losses occur at both electrodes in fuel cells, with its principle rooted in activation energy found in general chemical reactions. Since the activation loss at the anode side is much smaller compared to that at the cathode side for hydrogen PEMFC, only the latter is considered in this paper.

Equation (19) represent the activation overpotential (cathode only), derived from the Butler-Volmer equation, as described in (Zhang, 2012).

$$
\eta_{\text {act }}=\frac{R T}{4(1-\alpha) F} \ln \left[\frac{i+i_{\text {leak }}}{\left.i_{0}^{\text {ref }}\right|_{t=0}} \times \frac{1}{\xi_{\text {cat }}} \times\left(\frac{p_{O_{2}}^{\text {ref* }}}{p_{O_{2}}^{*}}\right)^{\alpha}\right]
$$

where $\alpha$ is the transfer coefficient and depends on the shape of the potential energy diagram that is specific to the oxygen 
reduction reaction, $i$ and $i_{\text {leak }}$ are the current density and the leak current density, $\left.i_{0}^{\text {ref }}\right|_{t=0}$ represents the reference exchange current density determined at the beginning of the fuel cell life, $\xi_{\text {cat }}$ represents the ratio of the actual catalyst surface area over the original catalyst surface area. This system parameter is slowly varying along with the gradual aging process that a fuel cell inevitable undergoes and is only a concern when long term operation issues are discussed. In this paper, $\xi_{\text {cat }}$ can be considered as a constant, since only short time scale problem is studied. $p_{O_{2}}^{\text {ref }}$ is the reference oxygen partial pressures at the reaction site, and can be determined experimentally.

\subsubsection{Ohmic Loss}

The overpotential due to Ohmic loss is simply based on the Ohmic law as follows

$$
\eta_{\text {ohm }}=i \cdot A_{\mathrm{fc}} \cdot R_{\text {ohm }}
$$

where $R_{\text {ohm }}$ is the total ohmic resistance of the fuel cell, electronic and ionic combined.

\subsubsection{Operating cell voltage}

By combining equations (17) - (20), we finally obtain the operating cell voltage as expressed in (21),

$$
\begin{aligned}
V_{\text {cell }}= & E_{0}\left(T_{\mathrm{fc}}\right)-\frac{R T_{\mathrm{fc}}}{4(1-\alpha) F} \ln \left(i+i_{\text {leak }}\right)+\frac{R T_{\mathrm{fc}}}{2 F} \ln p_{H_{2}}^{*} \\
& +\frac{R T_{\mathrm{fc}}}{4(1-\alpha) F} \ln p_{O_{2}}^{*}-i \cdot A_{\mathrm{fc}} \cdot R_{\text {ohm }}
\end{aligned}
$$

where $E_{0}\left(T_{\mathrm{fc}}\right)$ is the voltage component that only depends on the fuel cell temperature for a specific type of fuel cell. The cell voltage is regarded as a measured system output.

\subsubsection{Unified state space model}

The previously developed models are now summarized in the form of a unified state space model, combined with a new ODE capturing the $\mathrm{O}_{2}$ dynamics $\left(p_{c h}^{O_{2}}\right)$ in the channel, and represented by state equations (22) - (26) and output equations (27) - (28).

$$
\begin{array}{r}
\frac{M V_{s m}}{R T} \dot{p}_{s m}=\left(\dot{m}_{i n}^{a}+\dot{m}_{h m}^{v}\right)-\frac{p_{s m}-p_{c h}}{K_{s m, \text { out }}+K_{c h, i n}+\frac{k_{c h} L}{2}} \\
\frac{M V_{c h}}{R T} \dot{p}_{c h}=\frac{p_{s m}-p_{c h}}{K_{s m, o u t}+K_{c h, i n}+\frac{k_{c h} L}{2}-\frac{p_{c h}-p_{r m}}{\frac{k_{c h} L}{2}+K_{c h, o u t}+K_{r m, i n}}} \\
-D_{\text {eff }}\left(V_{G D L}^{l}\right) \cdot \frac{p_{c h}^{O_{2}}-p_{G D L}^{O_{2}}}{\delta_{G D L} / 2} \cdot A_{\mathrm{fc}} \cdot \frac{M_{O_{2}}}{R T}-\frac{M p_{c h}}{R T} \dot{V}_{c h}
\end{array}
$$

$$
\begin{aligned}
& \frac{M V_{r m}}{R T} \dot{p}_{r m}=\frac{p_{c h}-p_{r m}}{\frac{k_{c h} L}{2}+K_{c h, \text { out }}+K_{r m, \text { in }}}-\frac{p_{r m}-p_{b p r}}{K_{r m, \text { out }}} \\
& \frac{M_{O_{2}} V_{c h}}{R T} \dot{p}_{c h}^{O_{2}}=\frac{p_{s m}-p_{c h}}{K_{s m, \text { out }}+K_{c h, \text { in }}+\frac{k_{c h} L}{2}} \cdot \bar{x}^{a} x_{a}^{O_{2}} \\
& -\frac{p_{c h}-p_{r m}}{\frac{k_{c h} L}{2}+K_{c h, o u t}+K_{r m, i n}} \cdot \frac{M_{O_{2}} \cdot p_{c h}^{O_{2}}}{M \cdot p_{c h}} \\
& -D_{\text {eff }}^{O_{2}}\left(V_{G D L}^{l}\right) \cdot \frac{p_{c h}^{O_{2}}-p_{G D L}^{O_{2}}}{\delta_{G D L} / 2} \cdot A_{\mathrm{fc}} \cdot \frac{M_{O_{2}}}{R T} \\
& -\frac{M_{O_{2}} \cdot p_{c h}^{O_{2}}}{R T} \dot{V}_{c h} \\
& V_{G D L}^{p o r e} \dot{p}_{G D L}^{O_{2}}=D_{\text {eff }}^{O_{2}}\left(V_{G D L}^{l}\right) \cdot \frac{p_{c h}^{O_{2}}-p_{G D L}^{O_{2}}}{\delta_{G D L} / 2} \cdot A_{\mathrm{fc}}-\frac{W_{r c t}^{O_{2}}}{M_{O_{2}}} R T \\
& p_{c h, \text { in }}=p_{c h}+\left(\frac{p_{s m}-p_{c h}}{K_{s m, \text { out }}+K_{c h, \text { in }}+\frac{k_{c h} L}{2}}-W_{c 2 g}\right) \times\left(K_{c h, \text { in }}+\frac{k_{c h} L}{2}\right) \\
& V_{\text {cell }}=E_{0}\left(T_{\mathrm{fc}}\right)-\frac{R T_{\mathrm{fc}}}{4(1-\alpha) F} \ln \left(i+i_{\text {leak }}\right)-i \cdot A_{\mathrm{fc}} \cdot R_{\text {ohm }} \\
& +\frac{R T_{\mathrm{fc}}}{2 F} \ln p_{\mathrm{H}_{2}}^{*}+\frac{R T_{\mathrm{fc}}}{4(1-\alpha) F} \ln p_{\mathrm{O}_{2}}^{*}
\end{aligned}
$$

where $p^{* O_{2}}$ is given in(16), and $p_{H_{2}}^{*}=p_{H_{2}}^{c h}$ is assumed. $\bar{x}^{a}=1 / 1+\frac{p_{v}^{\text {sat }} M_{\mathrm{H}_{2} \mathrm{O}}}{M_{a}\left(p_{s m}-p_{v}^{\text {sat }}\right)}$ is the dry air mass fraction of the inlet humidified air. $x_{a}^{O_{2}}$ is the oxygen mass fraction in the environment air, which is given by $x_{a}^{O_{2}}=\frac{M_{O_{2}} \cdot 0.21}{M_{O_{2}} \cdot 0.21+M_{N_{2}} \cdot 0.79}$.

As can be seen in (22) and (27), the combined system is a fifth order dynamic system, which has two measurable outputs.

\section{A UKF-BASED FRAMEWORK}

\subsection{Bayesian framework for joint estimation}

We first consider a general joint estimation problem based on the following discrete system

$$
\begin{gathered}
\mathbf{x}_{k+1}=\mathbf{f}\left(\mathbf{x}_{k}, \mathbf{u}_{k}, \mathbf{v}_{k}, \mathbf{w}_{k}\right) \\
\mathbf{y}_{k}=h\left(x_{k}, u_{k}, n_{k}, w_{k}\right)
\end{gathered}
$$


where $\mathbf{x}_{\mathbf{k}}$ represent the states of the system, $\mathbf{y}_{\mathbf{k}}$ the outputs, $\mathbf{u}_{\mathbf{k}}$ the inputs $, \mathbf{v}_{\mathbf{k}}, \mathbf{n}_{\mathbf{k}}$ the process and measurement noises, respectively, and $\mathbf{w}_{\mathbf{k}}$ the time-varying system parameters. $\mathbf{f}$ is the state equation representing the system dynamics, which is generally nonlinear; and $\mathbf{h}$ is the measurement equation, in the form of a nonlinear map in general.

Since the parameters $\mathbf{w}_{\mathbf{k}}$ are unknown and time-varying, the state and parameter must be simultaneously and jointly estimated based on the noisy measured output. On the other hand, the dynamics of the time-varying system parameters are usually hard to describe, i.e., there is a lack of descriptive dynamic equation to characterize the parameters. A commonly used method to address this issue is to treat the parameter as a stochastic signal driven by a white noise $\left(\mathbf{r}_{\mathbf{k}}\right)$

$$
\mathbf{w}_{k+1}=\mathbf{w}_{k}+r_{k}
$$

Then, by concatenating the states and parameters to form an augmented state vector $\mathbf{x}_{\mathbf{k}}^{a}=\left[\begin{array}{ll}\mathbf{x}_{\mathbf{k}}^{T} & \mathbf{w}_{\mathbf{k}}^{T}\end{array}\right]^{T}$, joint state space equations (Wan \& Merwe, 2001) (assuming additive noises) can be obtained as follows

$$
\begin{aligned}
\mathbf{x}_{\mathbf{k}+1}^{a} & =\left[\begin{array}{c}
\mathbf{x}_{\mathbf{k}+1} \\
\mathbf{w}_{\mathbf{k}+1}
\end{array}\right]=\left[\begin{array}{c}
\mathbf{f}\left(\mathbf{x}_{\mathbf{k}}, \mathbf{u}_{\mathbf{k}}, \mathbf{w}_{\mathbf{k}}\right) \\
\mathbf{I} \cdot \mathbf{w}_{\mathbf{k}}
\end{array}\right]+\left[\begin{array}{c}
\mathbf{B} \cdot \mathbf{v}_{\mathbf{k}} \\
\mathbf{r}_{\mathbf{k}}
\end{array}\right] \\
& =\mathbf{F}\left(\mathbf{x}_{\mathbf{k}}^{a}, \mathbf{u}_{\mathbf{k}}\right)+\left[\begin{array}{c}
\mathbf{B} \cdot \mathbf{v}_{\mathbf{k}} \\
\mathbf{r}_{\mathbf{k}}
\end{array}\right] \\
\mathbf{y}_{\mathbf{k}} & =\mathbf{h}\left(\mathbf{x}_{\mathbf{k}}, \mathbf{u}_{\mathbf{k}}, \mathbf{w}_{\mathbf{k}}\right)+\mathbf{n}_{\mathbf{k}}=\mathbf{H}\left(\mathbf{x}_{\mathbf{k}}^{a}, \mathbf{u}_{\mathbf{k}}\right)+\mathbf{n}_{\mathbf{k}}
\end{aligned}
$$

The joint estimation problem of states and parameters based on observation can be formulated in an optimal recursive estimation framework as given in the following equation

$$
\hat{\mathbf{x}}_{\mathbf{k}}^{a}=E\left[\mathbf{x}_{\mathbf{k}}^{a} \mid \mathbf{Y}_{\mathbf{k}}\right]=E\left[\mathbf{x}_{\mathbf{k}}^{a} \mid \mathbf{y}_{\mathbf{0}}, \mathbf{y}_{\mathbf{1}}, \cdots \mathbf{y}_{\mathbf{k}}\right]
$$

Two step process (recursively) are involved, the first is the measurement correction

$$
p\left(\mathbf{x}_{\mathbf{k}}^{a} \mid \mathbf{Y}_{\mathbf{k}}\right)=\frac{p\left(\mathbf{x}_{\mathbf{k}}^{a} \mid \mathbf{Y}_{\mathbf{k}-1}\right) p\left(\mathbf{y}_{\mathbf{k}} \mid \mathbf{x}_{\mathbf{k}}^{a}\right)}{p\left(\mathbf{y}_{\mathbf{k}} \mid \mathbf{Y}_{\mathbf{k}-1}\right)}
$$

where $p($.$) indicates the probability density function.$

And the second is the one-step prediction

$$
p\left(\mathbf{x}_{\mathbf{k}+1}^{a} \mid \mathbf{Y}_{\mathbf{k}}\right)=\int p\left(\mathbf{x}_{\mathbf{k}+1}^{a} \mid \mathbf{x}_{\mathbf{k}}^{a}\right) p\left(\mathbf{x}_{\mathbf{k}}^{a} \mid \mathbf{Y}_{\mathbf{k}}\right) d \mathbf{x}_{\mathbf{k}}^{a}
$$

Various filtering techniques can be implemented in this general recursive estimation framework, including the most widely used extended Kalman filter (EKF), particle filtering (PF), and unscented Kalman filter (UKF). EKF is difficult to tune, and the Jacobian is usually hard to derive, and it can only handle limited amount of nonlinearity; while PF can handle arbitrary distributions and nonlinearities but is computationally very complex. In this paper, we focus on the UKF approach since we believe it gives a nice tradeoff between PF and EKF.

\subsection{UKF implementation}

We assume the additive (zero mean) noise case and follow the UKF procedure given in (Wan \& Merwe, 2001).

First, the augmented state estimation and covariance matrix are initialized with (37) and (38).

$$
\begin{gathered}
\hat{\mathbf{x}}_{0}^{a}=E\left[\mathbf{x}_{0}^{a}\right] \\
\mathbf{P}_{0}^{a}=E\left[\left(\mathbf{x}_{0}^{a}-\hat{\mathbf{x}}_{0}^{a}\right)\left(\mathbf{x}_{0}^{a}-\hat{\mathbf{x}}_{0}^{a}\right)^{T}\right]
\end{gathered}
$$

Then, for each iteration $(k=1,2, \ldots)$, the sigma points for the state variables in the last step are obtained and concatenated to form a matrix as follows

$$
\mathbf{X}_{k-1}=\left[\hat{\mathbf{x}}_{k-1}^{a}\left|\hat{\mathbf{x}}_{k-1}^{a}+c \sqrt{\mathbf{P}_{k-1}^{a}}\right| \hat{\mathbf{x}}_{k-1}^{a}-c \sqrt{\mathbf{P}_{k-1}^{a}}\right]
$$

where $\lambda=\alpha^{2}(L+\kappa)-L, c=L+\lambda, L$ is the dimension of the system given by (32), and $\alpha, \kappa$ and $\beta$ are all tunable parameters (in this paper, $\alpha=10^{-3}, \kappa=0, \beta=2$ is chosen according to (Wan \& Merwe, 2001)).

These sigma points are then fed to the state equation to generate a new set of sigma points for the state variables in the current step: $\mathbf{X}_{k \mid k-1}^{(i)}=\mathbf{F}\left(\mathbf{X}_{k-1}^{(i)}, \mathbf{u}_{\mathbf{k}}\right)$, where the superscript (i) denotes the $i$-th column of the corresponding matrix, i.e., the $i$-th sigma point, and $\mathbf{F}$ as in (32).

The one-step prediction for the augmented state vector in (36), when implemented with UKF approach, can now be expressed as

$$
\begin{gathered}
\hat{\mathbf{x}}_{k}^{a-}=\sum_{i=0}^{2 L} w_{i}^{m} \cdot \mathbf{X}_{k \mid k-1}^{(i)} \\
\mathbf{P}_{k}^{a-}=\sum_{i=0}^{2 L} w_{i}^{c} \cdot\left[\mathbf{X}_{k \mid k-1}^{(i)}-\hat{\mathbf{x}}_{k}^{a-}\right]\left[\mathbf{X}_{k \mid k-1}^{(i)}-\hat{\mathbf{x}}_{k}^{a-}\right]^{T}+\mathbf{Q}
\end{gathered}
$$

where $\mathbf{Q}$ is the process noise covariance matrix, and $w_{i}^{m}, w_{i}^{c}$ are the weights for the corresponding sigma points.

Measurement correction, on the other hand, is given through $(42) \sim(48)$

$$
\begin{gathered}
\mathbf{Y}_{k \mid k-1}^{(i)}=\mathbf{H}\left(\mathbf{X}_{k \mid k-1}^{(i)}, \mathbf{u}_{\mathbf{k}}\right) \\
\hat{\mathbf{y}}_{k}^{-}=\sum_{i=0}^{2 L} w_{i}^{\mathrm{m}} \cdot \mathbf{Y}_{k \mid k-1}^{(i)}
\end{gathered}
$$




$$
\begin{gathered}
\mathbf{P}_{\overline{\mathbf{y}}_{k} \overline{\mathbf{y}}_{k}}=\sum_{i=0}^{2 L} w_{i}^{\mathrm{c}} \cdot\left[\mathbf{Y}_{k \mid k-1}^{(i)}-\hat{\mathbf{y}}_{k}^{-}\right]\left[\mathbf{Y}_{k \mid k-1}^{(i)}-\hat{\mathbf{y}}_{k}^{-}\right]^{T}+\mathbf{R} \\
\mathbf{P}_{\overline{\mathbf{x}}_{k} \overline{\mathbf{y}}_{k}}=\sum_{i=0}^{2 L} w_{i}^{\mathrm{c}} \cdot\left[\mathbf{X}_{k \mid k-1}^{(i)}-\hat{\mathbf{x}}_{k}^{a-}\right]\left[\mathbf{Y}_{k \mid k-1}^{(i)}-\hat{\mathbf{y}}_{k}^{-}\right]^{T} \\
\mathbf{K}_{k}=\mathbf{P}_{\overline{\mathbf{x}}_{k} \overline{\mathbf{y}}_{k}} \mathbf{P}_{\overline{\mathbf{y}}_{k} \overline{\mathbf{y}}_{k}}^{-1} \\
\hat{\mathbf{x}}_{k}^{a}=\hat{\mathbf{x}}_{k}^{a-}+\mathbf{K}_{k}\left(\mathbf{y}_{k}-\hat{\mathbf{y}}_{k}^{-}\right) \\
\mathbf{P}_{k}^{a}=\mathbf{P}_{k}^{a-}-\mathbf{K}_{k} \mathbf{P}_{\overline{\mathbf{y}}_{k} \overline{\mathbf{y}}_{k}} \mathbf{K}_{k}^{T}
\end{gathered}
$$

where $\mathbf{R}$ is the measurement noise covariance matrix, and $\mathbf{H}$ as in (32).

The weights typically employed in UKF are given as follows

$$
w_{0}^{m}=\lambda / c, w_{0}^{c}=\lambda / c+\left(1-\alpha^{2}+\beta\right), w_{i}^{m}=w_{i}^{c}=\lambda / 2 c .
$$

\section{UKF DESIGN FOR FC FLOODING DIAGNOSTICS}

In this section, we design the diagnostic scheme for the fuel cell flooding based on the UKF framework presented in the last section. As will be discussed later in more details, when integrating the previously developed fuel cell model in the diagnostic scheme, the amounts of the liquid water in both the channel and the GDL are treated as varying system parameters (or unknown disturbances) to be identified, and modeled as stochastic processes driven by white noises, so that a standard joint estimation problem can then be formulated. The reason we treat these fault-related variables as system parameters, instead of building physics-based dynamic models for them and treating them as state variables, lies in the difficulty of modeling the water transport phenomena. On one hand, the dynamics of the channel water is highly stochastic and unpredictable, e.g., the water can accumulate to take up $40 \%$ of the channel volume (serious flooding) before being purged out, or it can get purged when only $10 \%$ of the channel volume (light flooding) is taken up due to the formation of a water film that obstructs the cross sectional area of the channel and totally shut off the passage of the streaming reactant gas. On the other hand, for the liquid water in the GDL, models have been developed that describe its transport driven by capillary pressure gradient ((D.A. McKay et al., 2005) and (Denise A. McKay et al., 2008)). However, since many factors can affect this phenomenon, (e.g., back diffusion of water from the cathode to the anode, and electro-osmotic drag that transports water from the anode to the cathode), and it's almost impossible to measure the related internal variables, such as the water concentration at the electrodes, it becomes very difficult to identify the model parameters involved. Therefore, in this paper, we consider both liquid water amounts in the GDL and the channel as stochastic processes.
As shown in Figure 5 (a), for decision making, the observer based diagnostic module is fed with: 1) the system inputs, i.e. the reactant mass flow rates controlled by the mass flow controllers (MFCs) at both sides; 2) the system outputs, i.e., the channel inlet pressures at both sides and the cell voltage; and 3) the current, which is regarded as the known disturbance to the system. With limited measurable outputs (three) and insufficient knowledge about the system dynamics (no model for liquid water transport dynamics), the problem of detecting and estimating four fault variables (anode channel and GDL flooding water, and cathode channel and GDL flooding water) becomes an unobservable one. Considering that the cathode GDL flooding has a much greater impact on the cell voltage than the same level anode GDL flooding (this conclusion can be obtained from model analysis), only cathode GDL flooding is considered in this paper while we assume no mass transport loss at anode, i.e., $p_{H_{2}}^{*}=p_{H_{2}}^{c h}$.

Now the diagnostic problem is reduced to detecting and estimating the flooding water in 1) anode channel, 2) cathode channel flooding, and 3) cathode GDL flooding. Since we have assumed the same partial pressure of $\mathrm{H}_{2}$ in the channel as that at the reaction site, the anode channel flooding diagnostic problem can be independently solved by utilizing only the input and output of the hydrogen supply system. Thus in this paper, we only focus on the design of the flooding diagnostic scheme at the fuel cell cathode side, whose model are represented by (22) - (28). To further simplify the problem, instead of applying the UKF based framework to the unified cathode model, we decompose the diagnostic scheme into two separate schemes respectively for the channel flooding and GDL flooding problems, as shown in Figure 5 (b). The two decomposed UKF based diagnostic schemes are now described in the following subsections.

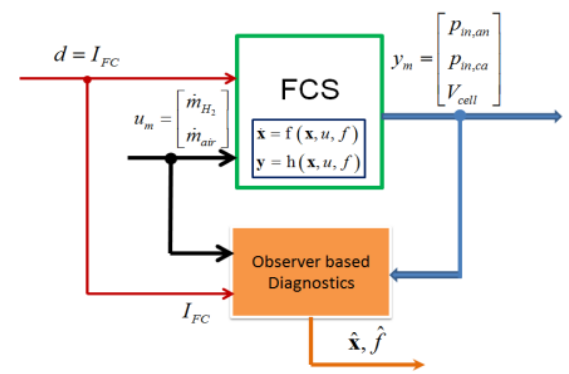

(a) 


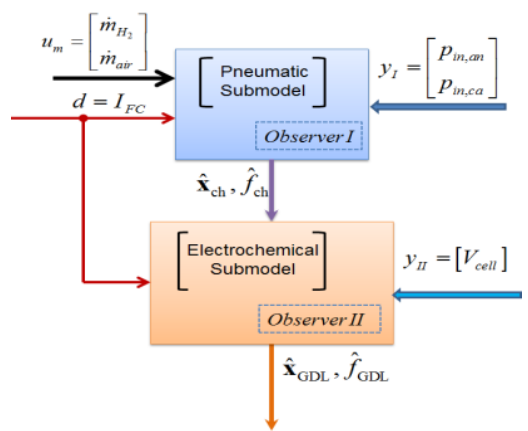

(b)

Figure 5 UKF based diagnostic scheme for fuel cell flooding fault (a) and its decomposition to dual observer-based diagnostic schemes (b)

\subsection{UKF design for channel flooding}

In model (22) - (28), the inlet pressure $p_{c h, i n}$ (equivalently the pressure drop, since the fuel cell back pressure $p_{b p r}$ is assumed to be a constant) is modeled as the subsystem's output. The impact of the channel flooding on this output variable is embodied by the term $k_{c h}=\frac{32 \mu(T) R T}{D_{h}^{2} \cdot A_{c h} \cdot M \cdot p_{c h}}$, which represents the friction in unit length against the gas flow in the channel. When water accumulates in the flow field, the channel cross sectional area $A_{c h}$ and the hydraulic diameter $D_{h}$ are equivalently reduced, resulting in an increased friction parameter $k_{c h}$, which in turn leads to a higher pressure drop. The formula above also reveals that $k_{c h}$ is inversely proportional to the square of the cross sectional area $A_{c h}$, since $D_{h}^{2}$ is proportional to $A_{c h}$.

To obtain a direct relationship between the friction parameter $k_{c h}$ and the amount of water accumulated in the channel (described by the water volume $V_{l, c h}$ ), the channel cross sectional area $A_{c h}$ is written as $A_{c h}=A_{c h}^{n o m}-\frac{V_{l, c h}}{L}=A_{c h}^{\text {nom }}\left(1-\frac{V_{l, c h}}{L \cdot A_{c h}^{n o m}}\right)$, where $A_{c h}^{\text {nom }}$ is the nominal value of the cross sectional area of the channel when no liquid water exists. This formula is based on the simplified assumption that the liquid water inside the channel is evenly spread along the length of the flow field. Note $D_{h}^{2}$ is proportional to $A_{c h}$, we then have

$D_{h}^{2} A_{c h}=\left(D_{h}^{n o m}\right)^{2} A_{c h}^{\text {nom }}\left(1-\frac{V_{l, c h}}{L \cdot A_{c h}^{\text {nom }}}\right)^{2}=\frac{\left(D_{h}^{n o m}\right)^{2} A_{c h}^{\text {nom }}}{\left(V_{c h}^{n o m}\right)^{2}}\left(V_{c h}\right)^{2}$.

Therefore, the term $\frac{k_{c h} L}{2}$ can be written as

$$
\frac{k_{c h} L}{2}=\frac{16 \mu(T) R T L\left(V_{c h}^{n o m}\right)^{2}}{M \cdot\left(D_{h}^{n o m}\right)^{2} A_{c h}^{n o m}} \frac{1}{p_{c h}\left(V_{c h}\right)^{2}}=\frac{\kappa_{\beta}}{p_{c h}\left(V_{c h}\right)^{2}}
$$

By substituting (49) in (7) and(10) while replacing $W_{\text {c2g }}$ with $W_{r c t}^{O_{2}}$, and also taking the derivative of $V_{c h}$, the model equations for the air supply system can now be rewritten as follows, with state equations represented by (50) - (52) and output equation represented by (53).

$$
\frac{M V_{s m}}{R T} \dot{p}_{s m}=\left(\dot{m}_{i n}^{a}+\dot{m}_{h m}^{v}\right)-\frac{p_{s m}-p_{c h}}{K_{s m, o u t}+K_{c h, \text { in }}+\frac{\kappa_{\beta}}{p_{c h}\left(V_{c h}\right)^{2}}}
$$

$$
\begin{aligned}
\frac{M V_{c h}}{R T} \dot{p}_{c h}= & \frac{p_{s m}-p_{c h}}{K_{s m, \text { out }}+K_{c h, \text { in }}+\frac{\kappa_{\beta}}{p_{c h}\left(V_{c h}\right)^{2}}} \\
& -\frac{p_{c h}-p_{r m}}{\frac{\kappa_{\beta}}{p_{c h}\left(V_{c h}\right)^{2}}+K_{c h, o u t}+K_{r m, i n}}-W_{r c t}^{O_{2}}-\frac{M p_{c h}}{R T} \dot{V}_{c h}
\end{aligned}
$$

$$
\frac{M V_{r m}}{R T} \dot{p}_{r m}=\frac{p_{c h}-p_{r m}}{\frac{\kappa_{\beta}}{p_{c h}\left(V_{c h}\right)^{2}}+K_{c h, o u t}+K_{r m, \text { in }}}-\frac{p_{r m}-p_{b p r}}{K_{r m, \text { out }}}
$$

$$
p_{c h, i n}=p_{c h}+\left(\frac{p_{s m}-p_{c h}}{K_{s m, o u t}+K_{c h, i n}+\frac{\kappa_{\beta}}{p_{c h}\left(V_{c h}\right)^{2}}}-W_{r c t}^{0_{2}}\right) \times\left(K_{c h, i n}+\frac{\kappa_{\beta}}{p_{c h}\left(V_{c h}\right)^{2}}\right)
$$

Note that, in order to decouple the channel flooding problem from the GDL flooding problem, approximation is made that $W_{\mathrm{c} 2 \mathrm{~g}}=W_{r c t}^{O_{2}}$. This is justified by the fact that the gas dynamics in the channel is much slower than that in the GDL due to the small GDL thickness.

The diagnostic problem is essentially an estimation problem for the water volume $V_{l, c h}$, or equivalently the channel volume $V_{c h}$, since $V_{c h}=L \cdot A_{c h}^{n o m}-V_{l, c h}=V_{c h}^{n o m}-V_{l, c h}$. While $V_{c h}$ appears in the state space equations as a varying parameter, it can be constructed as an observer problem simultaneously estimating the system state variables and parameters, using the UKF framework presented in Section 4. Therefore, the liquid water in the channel is considered as an unknown disturbance and its derivative is modeled as a process noise, i.e., $\dot{V}_{l, c h}=w_{l . c h}$. Since $V_{c h}$ is used as the parameter in (50) - (53), we obtain its derivative as $\dot{V}_{c h}=-\dot{V}_{l, c h}=-w_{l . c h}=w_{c h}$. 
InTERnAtional Journal of Prognostics AND HeAlth MANAGEMENT

To standardize the state-space model notation, let the states to be defined as $\left[\begin{array}{llll}p_{s m} & p_{c h} & p_{r m} & V_{c h}\end{array}\right]^{T}=$ $\left[\begin{array}{llll}x_{1} & x_{2} & x_{3} & x_{4}\end{array}\right]^{T}$, and the $\mathrm{O}_{2}$ reaction rate considered as a known disturbance $W_{r c t}^{O_{2}}=d_{r c t}$. Also note the total inlet air mass flow rate is $W_{i n}^{a}+W_{h m}^{v}=W_{i n}^{a}\left(1-x_{a m b}^{v}\right) \frac{1}{\bar{x}^{a}}$. As a consequence, we define this term as the control input, i.e., $\quad W_{i n}^{a}+W_{h m}^{v}=\left(1-x_{a m b}^{v}\right) \frac{1}{\bar{x}^{a}} W_{i n}^{a} \cdot b_{h m} \cdot u_{\mathrm{MF}} \quad, \quad$ where $b_{h m}\left(1-x_{a m b}^{v}\right) \frac{1}{\bar{x}^{a}}$ is a constant coefficient that only depends on the component of the ambient air and $u_{\mathrm{MF}} W_{i n}^{a}$ is the mass flow rate at the controller (MFC).

By substituting the above defined terms and the new state equation for the channel volume $\dot{V}_{c h}=w_{c h}$, equations (50) (53) are now modified to be (54) - (58)

$$
\begin{aligned}
& \dot{x}_{1}=-\alpha_{s m} \frac{x_{1}-x_{2}}{K_{n 1}+\frac{\kappa_{\beta}}{x_{2} x_{4}^{2}}}+\alpha_{s m} b_{h m} \cdot u_{\mathrm{MF}} \\
& \dot{x}_{2}=\frac{\theta_{M i x}}{x_{4}} \frac{x_{1}-x_{2}}{K_{n 1}+\frac{\kappa_{\beta}}{x_{2} x_{4}^{2}}}-\frac{x_{2}-x_{3}}{K_{n 2}+\frac{\kappa_{\beta}}{x_{2} x_{4}^{2}}}-\frac{\theta_{M i x}}{x_{4}} d_{r c t}-\frac{x_{2}}{x_{4}} w_{c h} \\
& \dot{x}_{3}=\alpha_{r m} \frac{x_{2}-x_{3}}{K_{n 2}+\frac{\kappa_{\beta}}{x_{2} x_{4}^{2}}}-\alpha_{r m} \frac{x_{3}-p_{b p r}}{K_{3}} \\
& \dot{x}_{4}=w_{c h} \\
& y=x_{2}+\left(\frac{x_{1}-x_{2}}{K_{n 1}+\frac{\kappa_{\beta}}{x_{2} x_{4}^{2}}}-d_{r c t}\right) \times\left(K_{c h, i n}+\frac{\kappa_{\beta}}{x_{2} x_{4}^{2}}\right)+v_{m}
\end{aligned}
$$

where $\quad \alpha_{s m}=\frac{R T}{M V_{s m}}, \alpha_{r m}=\frac{R T}{M V_{r m}}$, and $\theta_{M i x}=\frac{R T}{M}$. $K_{n 1}=K_{s m, \text { out }}+K_{c h, \text { in }}, K_{n 2}=K_{c h, o u t}+K_{r m, \text { in }}, K_{3}=K_{r m, o u t} \cdot v_{m}$ is the measurement noise which is treated as white noise here.

To apply the discrete UKF approach, the system needs to be discretized first. Using the forward Euler method and sampling time $\Delta T$, we obtain the following discrete state space equations as given in (59) (63).

$$
x_{1, k+1}=x_{1, k}-\Delta T \cdot \alpha_{s m} \frac{x_{1, k}-x_{2, k}}{K_{n 1}+\frac{\kappa_{\beta}}{x_{2, k} x_{4, k}^{2}}}+\Delta T \cdot \alpha_{s m} b_{h m} \cdot u_{\mathrm{MF}, k}
$$

$$
\begin{gathered}
x_{2, k+1}=x_{2, k}+\Delta T \cdot \frac{\theta_{M i x}}{x_{4, k}} \frac{x_{1, k}-x_{2, k}}{K_{n 1}+\frac{\kappa_{\beta}}{x_{2, k} x_{4, k}^{2}}}-\Delta T \cdot \frac{x_{2}}{x_{4}} w_{c h, k} \\
-\Delta T \cdot \frac{x_{2, k}-x_{3, k}}{K_{n 2, k}+\frac{\kappa_{\beta}}{x_{2, k} x_{4, k}^{2}}-\Delta T \cdot \frac{\theta_{M i x}}{x_{4, k}} d_{r c t, k}} \\
x_{3, k+1}=x_{3, k}+\Delta T \cdot \alpha_{r m} \frac{x_{2, k}-x_{3, k}}{K_{n 2, k}+\frac{\kappa_{\beta}}{x_{2, k} x_{4, k}^{2}}-\Delta T \cdot \alpha_{r m} \frac{x_{3, k}-p_{b p r}}{K_{3}}} \\
y_{k}=x_{2, k}+\left(\begin{array}{c}
x_{4, k+1}=x_{4, k}+\Delta T \cdot w_{c h, k} \\
\frac{x_{1, k}-x_{2, k}}{K_{n 1}+\frac{\kappa_{\beta}}{x_{2, k} 2_{4, k}^{2}}}-d_{r c t, k}
\end{array}\right) \times\left(K_{c h, i n}+\frac{\kappa_{\beta}}{x_{2, k} x_{4, k}^{2}}\right)+v_{m, k}
\end{gathered}
$$

The standard UKF procedure can then be applied to estimate the state vector $\left[\begin{array}{llll}x_{1, k} & x_{2, k} & x_{3, k} & x_{4, k}\end{array}\right]^{T}$ in the above discrete system.

\subsection{UKF design for GDL flooding}

The UKF-based diagnostic scheme for the GDL flooding diagnosis can be designed in a similar way to that of the channel flooding. As shown in Figure 6, the diagnostic submodule is based on the gas dynamic model in the GDL and the cell voltage model, and is fed with the current, the voltage, and as well as the estimated states and parameter from the channel flooding diagnostic sub-module.

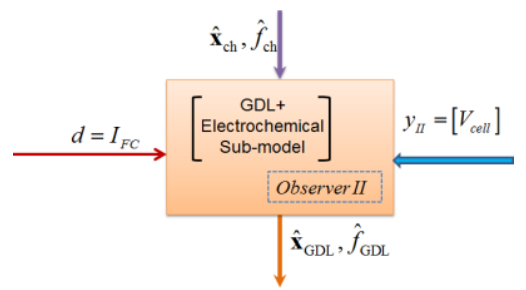

Figure 6 UKF based diagnostic scheme for the cathode GDL flooding

The state space model formulated in the standard simultaneous estimation of state and parameter problem is presented as follows: 


$$
\begin{aligned}
& \frac{M_{O_{2}} V_{c h}}{R T} \dot{p}_{c h}^{O_{2}}=\frac{\hat{p}_{s m}-\hat{p}_{c h}}{K_{s m, \text { out }}+K_{c h, \text { in }}+\frac{\kappa_{\beta}}{\hat{p}_{c h}\left(\hat{V}_{c h}\right)^{2}}} \cdot \bar{x}^{a} x_{a}^{O_{2}} \\
& -\frac{\hat{p}_{c h}-\hat{p}_{r m}}{\frac{\kappa_{\beta}}{\hat{p}_{c h}\left(\hat{V}_{c h}\right)^{2}}+K_{c h, \text { out }}+K_{r m, \text { in }}} \cdot \frac{M_{O_{2}} \cdot p_{c h}^{O_{2}}}{M \cdot \hat{p}_{c h}} \\
& -D_{\text {eff }}\left(V_{G D L}^{l}\right) \cdot \frac{p_{c h}^{O_{2}}-p_{G D L}^{O_{2}}}{\delta_{G D L} / 2} \cdot A_{\mathrm{fc}} \cdot \frac{M_{O_{2}}}{R T}+w_{c h}^{O_{2}} \\
& V_{G D L}^{p o r e} \dot{p}_{G D L}^{O_{2}}=D_{\text {eff }}\left(V_{G D L}^{l}\right) \cdot \frac{p_{c h}^{O_{2}}-p_{G D L}^{O_{2}}}{\delta_{G D L} / 2} \cdot A_{\mathrm{fc}}-\frac{W_{r c t}^{O_{2}}}{M_{O_{2}}} R T \\
& \dot{V}_{G D L}^{l}=w_{G D L}(t) \\
& p^{* O_{2}}=p_{G D L}^{O_{2}}-W_{r c t}^{O_{2}} \frac{\delta_{G D L} / 2 \cdot R T}{M_{O_{2}} A_{\mathrm{fc}} D_{\text {eff }}\left(V_{G D L}^{l}\right)} \\
& V_{\text {cell }}=E_{0}\left(T_{\mathrm{fc}}\right)-\frac{R T_{\mathrm{fc}}}{4(1-\alpha) F} \ln \left(i+i_{\text {leak }}\right)-i \cdot A_{\mathrm{fc}} \cdot R_{\text {ohm }} \\
& +\frac{R T_{\mathrm{fc}}}{2 F} \ln p_{\mathrm{H}_{2}}^{*}+\frac{R T_{\mathrm{fc}}}{4(1-\alpha) F} \ln p_{\mathrm{O}_{2}}^{*}
\end{aligned}
$$

where $w_{c h}^{O_{2}}$ is a white process noise that can be used to account for the estimation error from the first observer UKF I. Again, the system can then be discretized, standardized in notation, and employed by the standard UKF diagnostic scheme to jointly estimate $p_{c h}^{O_{2}}, p_{G D L}^{O_{2}}$, and most importantly $V_{G D L}^{l}$.

\section{SIMULATION RESULTS}

To validate the proposed flooding diagnosis scheme, simulation is carried out under constant current mode, with the simulation results presented in Figure 9-Figure 11.

The stepwise current profile for this simulation and the corresponding voltage response are shown in Figure 7, where the ripples of the voltage plot is caused by the varying flooding levels that is considered to be fault in the fuel cell. The faults are injected into the system by dictating the system parameters, i.e. the liquid water volumes in the channel and the GDL, to follow certain preset profiles as shown in Figure 8. The dictated channel water volume follows a zigzag like profile with a period of 300 seconds, emulating the sudden purge and the gradual accumulating process of the liquid water in the channel; while the dictated GDL water volume varies in a small range around some steady states that correspond to different levels of current loads, reflecting the trend of more serious flooding under larger current loads due to more water being produced. The steady state levels of the GDL water volume corresponds roughly to the simulation results using a more complex, detailed fuel cell dynamic model. Each dictated water volume is superimposed by a stochastic process driven by white noise, with the noise power being $\left(V \cdot 10^{-3}\right)^{2}$, where $V$ is the corresponding volume (channel, or GDL).

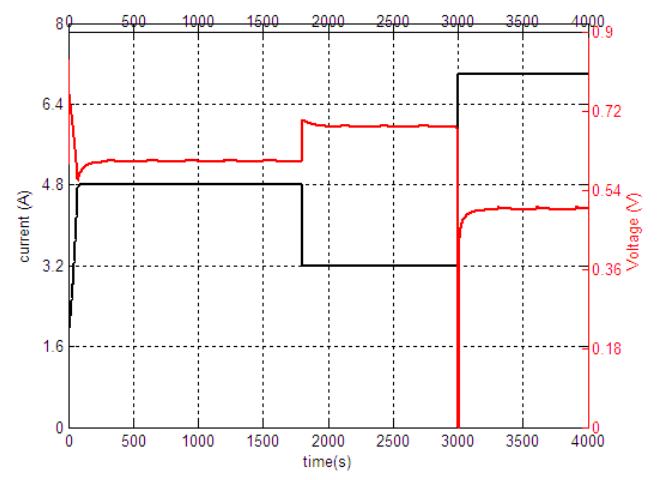

Figure 7 Current profile used in the simulation and the corresponding voltage in response
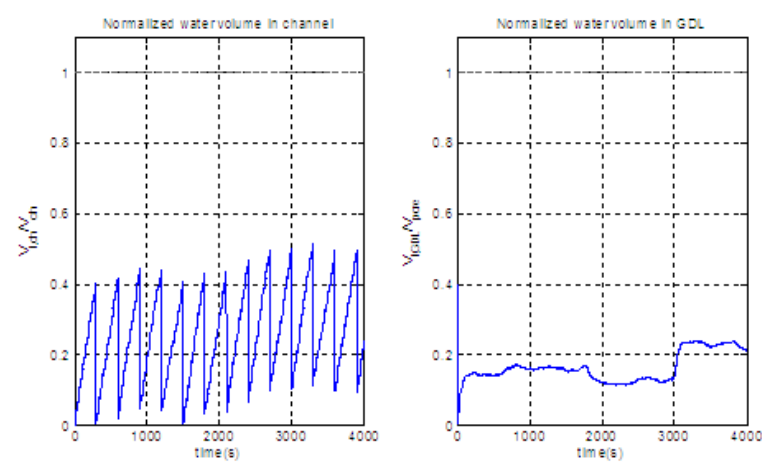

Figure 8 Dictated water volumes (normalized) in the channel and the GDL of the cathode

It can be seen from Figure 9 and Figure 10 that, by carefully choosing the observer parameters, UKF I successfully tracks the system parameter (the channel volume) and the state variables (the total pressures in various separate volumes of the fuel cell air supply system). The actual and estimated values for the states and parameters overlap almost perfectly with the given scale in the plots. Note that, this accuracy is achieved by assuming exact knowledge of the deterministic part of the system model, otherwise larger estimation errors are expected to be present due to the modeling error. In this paper, since our purpose is mainly to prove the concept and applicability of the cascading UKFs framework for joint state and parameter estimation on the fuel cell flooding diagnostic problem, exact model knowledge is thus assumed. 


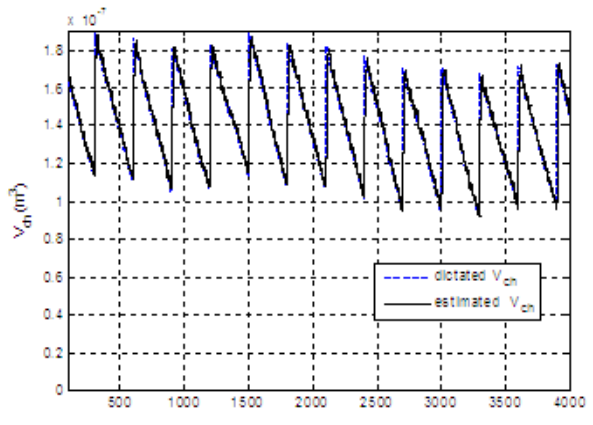

Figure 9 Simulation results by Observer I: Dictated and estimated channel volumes (cathode)

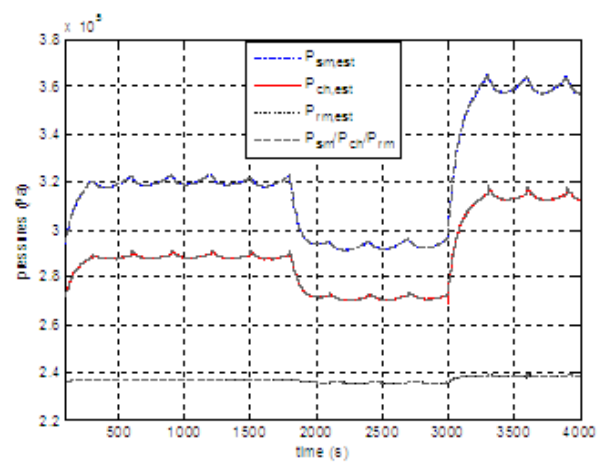

Figure 10 Simulation results by Observer I: Actual and estimated total pressures in various separate volumes of the fuel cell air supply system

The estimation result from the second UKF, however, is less accurate as shown in Figure 11. Although the estimated water volume follows the general trend of the actual flooding level, two discernible types of mismatches can be seen in the plot, the first being the zigzag like fluctuation around the steady states, and the second being the sudden drop of the estimated value at 3000 s when the flooding level in the GDL starts to increase.

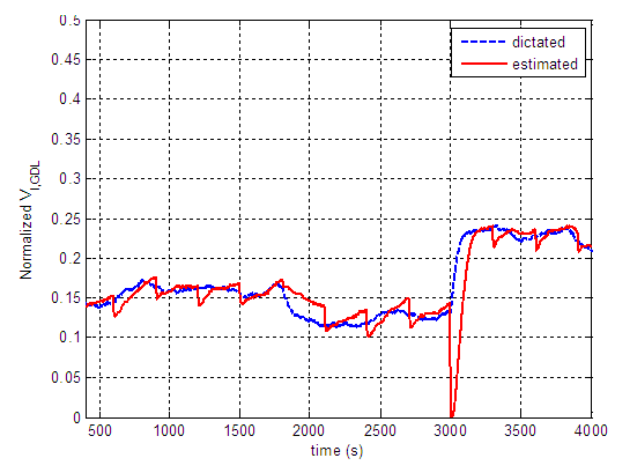

Figure 11 Simulation result by Observer II: Dictated and estimated water volume in the cathode GDL

A closer look into the problem reveals that the two types of mismatches actually both correspond to the sudden "purge" of the channel water (refer to Figure 8), which leads to a large estimation error of the oxygen partial pressure in the channel when occurs. By revisiting Figure 6 and equation(64), it can be seen that, due to the decomposition of the unified system model (22), the estimated states $\left(\hat{p}_{s m}, \hat{p}_{c h}, \hat{p}_{r m}\right)$ and parameter $\left(\hat{V}_{c h}\right)$ from UKF I are utilized as observer inputs in UKF II. Therefore, any estimation error by UKF I could be passed on to UKF II and affect its estimation results.

In equation(64), we have incorporated the additive process noise $w_{c h}^{O_{2}}$ as a mechanism to account for the estimation error from UKF I (a simplified method since the overall effect of all the estimation errors may be non-additive). While smaller variance values for the process noise $w_{c h}^{O_{2}}$ result in more rapid tracking of the system parameter $V_{G D L}^{l}$ due to relatively larger variance values for $w_{G D L}(t)$, the estimated results are also more susceptible to the estimation error from UKF I (e.g. Figure 11); on the other hand, with larger variance values for $w_{c h}^{O_{2}}$, UKF II becomes less sensitive to the estimation error from UKF I since less confidence is placed on the gas dynamics in the channel, but also becomes less responsive in tracking $V_{G D L}^{l}$.

To address this issue, adjustable variance for the process noise $w_{c h}^{O_{2}}$ is adopted in this paper. Since large estimation errors in UKF I mainly happen when the channel water gets purged out of the fuel cell, covariance for $w_{c h}^{O_{2}}$ is switch to larger value (less confidence) at these sudden "purges" that can be indicated by abruptly increased error between estimated and measured voltage output, and remains to be small for more accurate tracking of the GDL water volume when the parameter is varying in a much slower fashion compared to a purge. The switching rule of the variance value for $w_{c h}^{O_{2}}$ can be expressed as follows

$$
Q_{c h}^{O_{2}}=\left\{\begin{array}{l}
Q_{1} \quad \text { when } \mathrm{H}\left(\left|\frac{1}{T_{V} s+1} V_{\text {cell }}-\hat{V}_{\text {cell }}\right|\right)=0 \\
Q_{2} \quad \text { when } \mathrm{H}\left(\left|\frac{1}{T_{V} s+1} V_{\text {cell }}-\hat{V}_{\text {cell }}\right|\right)=1
\end{array}\right.
$$

where $\mathrm{H}(\bullet)$ is a sharp (square) hysteresis function, with $\Delta V_{\text {cell,th } 1}$ and $\Delta V_{\text {cell,th } 2}$ as the thresholds, 1 and 0 as the outputs. To eliminate the effect of the signal noise, the measured cell voltage output is filtered before being compared to the estimated voltage by a low pass filter with a time constant $T_{V}$.

Figure 12 shows the simulation result by Observer II with adjustable process noise $w_{c h}^{O_{2}}$. It can be seen that the sudden 
jumps in the estimated $V_{G D L}^{l}$ corresponding to the sudden purges of the channel water are now eliminated.

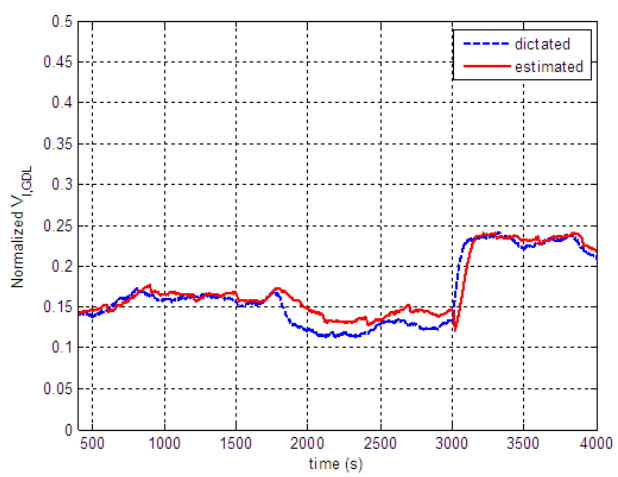

Figure 12 Simulation results by Observer II: Dictated and estimated water volume in the cathode GDL with adjustable process noise

The actual and estimated oxygen partial pressure in the GDL are compared in Figure 13, where the spikes every 300 s correspond to the purges of the channel water. It is seen that the UKF II is able to track the state variable fairly accurately except shortly (about 20s) after the purge occurs. This can be explained by the larger variance value assigned to $w_{c h}^{O_{2}}$, which makes the process noise for the GDL gas dynamics comparatively small, and thus leads to a slower tracking for this state variable $\left(\dot{p}_{G D L}^{O_{2}}\right)$.

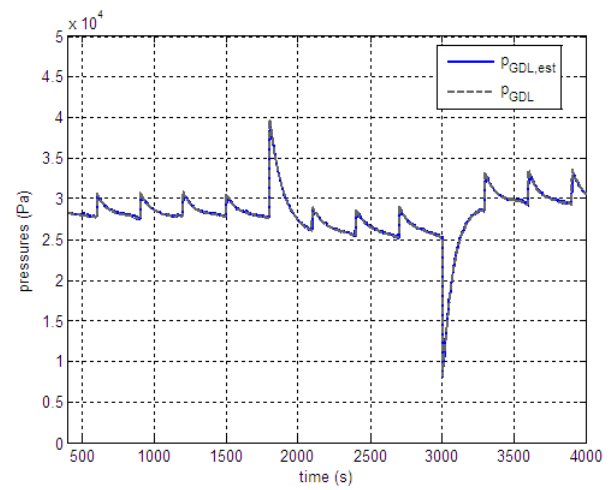

(a)

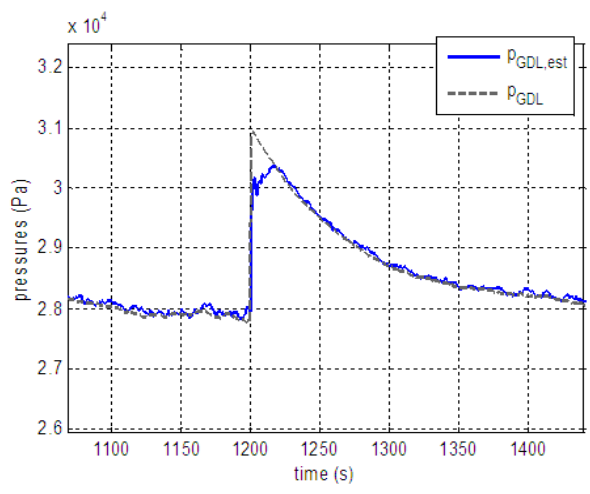

(b)

Figure 13 Simulation results by Observer II: (a) Actual and estimated oxygen partial pressures in the GDL with adjustable process noise; (b) close-up of (a)

\section{SUMMARY AND DISCUSSION}

In this paper, a cascaded UKF framework is proposed for the diagnosis of a PEM fuel cell flooding problem. A lumped fuel cell model is developed to incorporate the fault effect on the system dynamics by exploiting the relationship between the accumulated flooding water and the frictional resistance to the gas mixture flow in the channel, and that between the GDL liquid water and the reactant diffusivity through the gas diffusion media. Since the water dynamics in a fuel cell is highly stochastic and difficult to characterize, simple stochastic processes driven by white noises are used to model the volumes of the flooding water, which are treated as varying system parameters to facilitate the standard formulation of nonlinear state and parameter joint estimation problem to which the UKF technique can be applied.

For hydrogen type fuel cell with open ends on both anode and cathode sides, the GDL flooding is more likely to happen at the cathode than the anode. The cathode GDL flooding also has a much greater impact on the cell voltage than the same level of anode GDL flooding. By approximating the hydrogen partial pressure at the catalyst site with the estimated hydrogen partial pressure in the anode channel, we are able to focus on the flooding diagnosis at the cathode side only (in the channel and in the GDL). Further, by exploiting the time scale difference between the GDL and the channel modules, the two subsystems are decoupled from each other, facilitating the independent design of the observers for the two subsystems. However, the decoupling also leads to large estimation discrepancy in the second observer due to its dependence on the estimation results of the first observer. Adjustable variance for the process noise $w_{c h}^{O_{2}}$ is employed in this paper and is demonstrated to have successfully eliminated the major estimation error in observer II. 
It should be noted that since the purpose of this paper is mainly to prove the concept and applicability of the cascading UKFs framework for joint state and parameter estimation on the fuel cell flooding diagnostic problem, exact model knowledge has been assumed. Therefore, estimation error due to the modeling error is not considered here. Also, since "fully humidified inlet reactant" is among the main assumptions, drying condition has not been considered in the paper. The inclusion of the drying as a faulty condition in the fault set together with the GDL flooding and the channel flooding would again, not surprisingly, render the problem unobservable under the circumstances that no further information is known with regard to the water dynamics in the GDL. To address this issue and distinguish between membrane drying and GDL flooding, two approaches may be explored. One is to model the GDL water dynamics and incorporate it into our diagnosis-oriented fuel cell model; the other is to measure the high frequency response of the fuel cell impedance, which is essentially the same to obtain one point (in the high frequency region) at the EIS of the fuel cell. Note the latter approach is not intrusive as it dictates no operating condition change and can be carried out by imposing a very small current or voltage perturbation at a fixed (high) frequency.

\section{REFERENCES}

Aitouche, A., Yang, Q., Ould Bouamama, B., Aitouche, A., Yang, Q., \& Ould Bouamama, B. (2011). Fault detection and isolation of PEM fuel cell system based on nonlinear analytical redundancy: An application via parity space approach. EPJ Applied Physics, 54(2). doi:10.1051/epjap/2011100250

Arcak, M., Gorgun, H., Pedersen, L. M., \& Varigonda, S. (2004). A Nonlinear Observer Design for Fuel Cell Hydrogen Estimation. IEEE Transactions on Control Systems Technology, 12(1), 101-110. doi:10.1109/TCST.2003.821958

Barbir, F., Gorgun, H., \& Wang, X. (2005). Relationship between pressure drop and cell resistance as a diagnostic tool for PEM fuel cells. Journal of Power Sources, $\quad 141(1)$, 96-101. doi:10.1016/j.jpowsour.2004.08.055

Bosco, A. D., \& Fronk, M. H. (2000). Fuel cell flooding detection and correction.

Chen, J., \& Zhou, B. (2008). Diagnosis of PEM fuel cell stack dynamic behaviors. Journal of Power Sources, 177(1), 83-95.

Danzer, M. A., Wilhelm, J., Aschemann, H., \& Hofer, E. P. (2008). Model-based control of cathode pressure and oxygen excess ratio of a PEM fuel cell system. Journal of Power Sources, 176(2), 515-522.

Fouquet, N., Doulet, C., Nouillant, C., Dauphin-Tanguy, G., \& Ould-Bouamama, B. (2006). Model based PEM fuel cell state-of-health monitoring via ac impedance measurements. Journal of Power Sources, 159(2), 90513. doi:10.1016/j.jpowsour.2005.11.035

Gazzarri, J., Eikerling, M., Qianpu Wang, \& Zhong-Sheng Liu. (2010). Estimation of Local Relative Humidity in Cathode Catalyst Layers of PEFC. Electrochemical and Solid-State Letters, 13(6), 58-62. doi:10.1149/1.3355233

Gebregergis, A., Pillay, P., \& Rengaswamy, R. (2010). PEMFC fault diagnosis, modeling, and mitigation (Vol. 46, pp. 295-303). 445 Hoes Lane / P.O. Box 1331, Piscataway, NJ 08855-1331, United States: Institute of Electrical and Electronics Engineers Inc. doi:10.1109/TIA.2009.2036677

Gorgun, H., Arcak, M., \& Barbir, F. (2006). An algorithm for estimation of membrane water content in PEM fuel cells. Journal of Power Sources, 157(1), 389-394. doi:10.1016/j.jpowsour.2005.07.053

Hissel, D., Péra, M., \& Kauffmann, J. (2004). Diagnosis of automotive fuel cell power generators. Journal of Power Sources, 128(2), 239-246.

Hoshiko, T., Nakajima, H., Konomi, T., Kitahara, T., \& Kita, S. (2008). Estimation of Water Layer Thickness Adjacent to the Cathode Catalyst Layer of a PEFC (Analysis Using Electrochemical Impedance Spectroscopy) (Vol. 16, pp. 2117-2123). ECS. doi:10.1149/1.2982051

Kadyk, T., Hanke-Rauschenbach, R., \& Sundmacher, K. (2009). Nonlinear frequency response analysis of PEM fuel cells for diagnosis of dehydration, flooding and CO-poisoning. Journal of Electroanalytical Chemistry, 630(1-2), 19-27.

Kumbur, E. C., Sharp, K. V., \& Mench, M. M. (2006). Liquid droplet behavior and instability in a polymer electrolyte fuel cell flow channel. Journal of Power Sources, 161(1), 333-45.

Le Canut, J.-M., Abouatallah, R. M., \& Harrington, D. A. (2006). Detection of Membrane Drying, Fuel Cell Flooding, and Anode Catalyst Poisoning on PEMFC Stacks by Electrochemical Impedance Spectroscopy. Journal of The Electrochemical Society, 153(5), A857. doi:10.1149/1.2179200

Makharia, R., Mathias, M. F., \& Baker, D. R. (2005). Measurement of Catalyst Layer Electrolyte Resistance in PEFCs Using Electrochemical Impedance Spectroscopy. Journal of The Electrochemical Society, 152(5), A970. doi:10.1149/1.1888367

McKay, D., \& Stefanopoulou, A. (2004). Parameterization and validation of a lumped parameter diffusion model for fuel cell stack membrane humidity estimation. In American Control Conference, 2004. Proceedings of the 2004 (Vol. 1, pp. 816-821). Retrieved from http://ieeexplore.ieee.org/xpls/abs_all.jsp?arnumber=13 83706

McKay, D.A., Ott, W. T., \& Stefanopoulou, A. G. (2005). Modeling, parameter identification, and validation of reactant and water dynamics for a fuel cell stack. In 
2005 ASME International Mechanical Engineering Congress and Exposition, IMECE 2005, November 5, 2005 - November 11, 2005 (Vol. 74 DSC, pp. 11771186). Orlando, FL, United states: American Society of Mechanical Engineers.

McKay, Denise A., Siegel, J. B., Ott, W., \& Stefanopoulou, A. G. (2008). Parameterization and prediction of temporal fuel cell voltage behavior during flooding and drying conditions. Journal of Power Sources, 178(1), 207-222.

Narjiss, A., Depernet, D., Candusso, D., Gustin, F., \& Hissel, D. (2008). Online diagnosis of PEM fuel cell. In 2008 13th International Power Electronics and Motion Control Conference, EPE-PEMC 2008, September 1, 2008 - September 3, 2008 (pp. 734-739). Poznan, Poland: Inst. of Elec. and Elec. Eng. Computer Society. doi:10.1109/EPEPEMC.2008.4635354

Niroumand, A. M., Merida, W., \& Saif, M. (2011). PEM fuel cell low flow FDI. Journal of Process Control, 21(4), 602-612. doi:10.1016/j.jprocont.2010.12.013

O'Hayre, R., Cha, S.-W., Colella, W., \& Prinz, F. B. (2009). Fuel Cell Fundamentals (2nd ed.). Wiley.

Pei, P., Ouyang, M., Feng, W., Lu, L., Huang, H., \& Zhang, J. (2006). Hydrogen pressure drop characteristics in a fuel cell stack. International Journal of Hydrogen Energy, 31(3), 371-377.

Pukrushpan, J. T., Peng, H., \& Stefanopoulou, A. G. (2004). Control-Oriented Modeling and Analysis for Automotive Fuel Cell Systems. Journal of Dynamic Systems, Measurement, and Control, 126(1), 14. doi:10.1115/1.1648308

Steiner, N. Yousfi, Candusso, D., Hissel, D., \& Mooteguy, P. (2010). Model-based diagnosis for proton exchange membrane fuel cells (Vol. 81, pp. 158-170). P.O. Box 211, Amsterdam, 1000 AE, Netherlands: Elsevier. doi:10.1016/j.matcom.2010.02.006

Steiner, N.Y., Hissel, D., Mocoteguy, P., \& Candusso, D. (2011). Non intrusive diagnosis of polymer electrolyte fuel cells by wavelet packet transform. International Journal of Hydrogen Energy, 36(1), 740-6. doi:10.1016/j.ijhydene.2010.10.033

Vepa, R. (2012). Adaptive State Estimation of a PEM Fuel Cell. IEEE Transactions on Energy Conversion, 27(2), 457-467. doi:10.1109/TEC.2012.2190073

Wan, E., \& Merwe, R. (2001). Chapter 7: The Unscented Kalman Filter. In Kalman Filtering and Neural Networks. Wiley Publishing.

Wasterlain, S., Harel, F., Candusso, D., Hissel, D., \& Francois, X. (2009). First results obtained with an impedance meter developed for the diagnosis of large Proton-Exchange-Membrane Fuel-Cell stacks. In Advanced Electromechanical Motion Systems \& Electric Drives Joint Symposium, 2009. ELECTROMOTION 2009. 8th International Symposium on (pp. 1-6).
Wu, J., Yuan, X. Z., Wang, H., Blanco, M., Martin, J. J., \& Zhang, J. (2008). Diagnostic tools in PEM fuel cell research: Part I Electrochemical techniques. International Journal of Hydrogen Energy, 33(6), 1735-1746.

Xiaozi Yuan, Haijiang Wang, Jian Colin Sun, \& Jiujun Zhang. (2007). AC impedance technique in PEM fuel cell diagnosis - a review. International Journal of Hydrogen Energy, 32(17), 4365-80. doi:10.1016/j.ijhydene.2007.05.036

Yuan, X., Sun, J. C., Wang, H., \& Zhang, J. (2006). AC impedance diagnosis of a $500 \mathrm{~W}$ PEM fuel cell stack. Part II: Individual cell impedance. Journal of Power Sources, 161(2), 929-937. doi:10.1016/j.jpowsour.2006.07.020

Zhang, X. (2012). Prognostic and Health-Management Oriented Fuel Cell Modeling and On-line Supervisory System Development (Dissertation). Clemson University.

Zhang, X., \& Pisu, P. (2012). An Unscented Kalman Filter Based Approach for the Health-Monitoring and Prognostics of a PEM Fuel Cell (Vol. 3). Presented at the Annual Conference of the Prognostics and Health Management Society 2012, Minneapolis, MN.

Zhang, X., \& Pisu, P. (2014). Prognostic-oriented Fuel Cell Aging Modeling and Its Application to HealthMonitoring and Prognostics of a PEM Fuel Cell. International Journal of Prognostics and Health Management 1(5).

\section{BIOGRAPHIES}

Xian Zhang received his B.S. and M.S. degrees in Automotive Engineering from Tsinghua University, Beijing, China, in 2005 and 2007, respectively, and received his Ph.D. degree in Automotive Engineering from Clemson University, Clemson, SC in August 2012. His Ph.D. research area is in the control, diagnostics and prognostics of the PEMFC system. From September 2007 to May 2012, he was a Graduate Research Assistant with the International Center for Automotive Research and Department of Automotive Engineering, Clemson University. From June to November 2010, he was an intern with National Transportation Research Center (NTRC), at Oak Ridge National Lab. Xian Zhang is a student member of ASME and SAE.

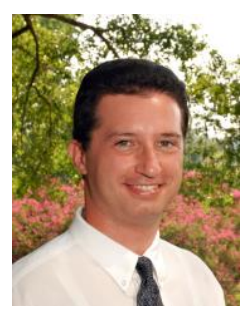

Pierluigi Pisu is an Assistant Professor at the Department of Automotive Engineering and holds a joint appointment with the Department of Electrical and Computer Engineering at Clemson University. He received his Ph.D. in Electrical Engineering from the Ohio State University in 2002. In 2004, Dr. Pisu was granted two US patents in area of model-based 
fault detection and isolation. Dr. Pisu's research interests are in the area of fault diagnosis and prognosis with application to vehicle systems, energy management control of hybrid electric vehicles, vehicle to grid interaction, and systems aging. He also worked in the area of sliding mode and robust control. Dr. Pisu is member of IEEE, ASME and SAE. He is member of the IEEE Technical Committee in Automotive Control, IFAC Technical Committee in Automotive Control, Associate Editor of the Conference Editorial Board of the IEEE Control Systems Society, and Associate Editor of the SAE Journal on Alternative Powertrains. 